\title{
Hypersurfaces with free boundary and large constant mean curvature: concentration along submanifolds
}

\author{
Mouhamed Moustapha Fall and Fethi Mahmoudi
}

\begin{abstract}
Given a domain $\Omega$ of $\mathbb{R}^{m+1}$ and a $k$-dimensional non-degenerate minimal submanifold $K$ of $\partial \Omega$ with $1 \leq k \leq m-1$, we prove the existence of a family of embedded constant mean curvature hypersurfaces in $\Omega$ which as their mean curvature tends to infinity concentrate along $K$ and intersecting $\partial \Omega$ perpendicularly along their boundaries.
\end{abstract}

Mathematics Subject Classification (2000): 53A10 (primary); 53C21, 35R35 (secondary).

\section{Introduction}

Let $\Omega$ be an open bounded subset of $\mathbb{R}^{m+1}, m \geq 2$, with smooth boundary $\partial \Omega$. Recall that the partitioning problem in $\Omega$ consists on finding, for a given $0<v<$ meas $(\Omega)$, a critical point of the perimeter functional $\mathcal{P}(\cdot, \Omega)$ in the class of sets in $\Omega$ that enclose a volume $v$. Here $\mathcal{P}(E, \Omega)$ denotes the perimeter of $E$ relative to $\Omega$.

It is clear that whenever such a surface exits it will meet $\partial \Omega$ orthogonally and will have a constant mean curvature, see Paragraph 2.3.1. In the light of standard results in geometric measure theory, minimizers do exist for any given volume and may have various topologies (see the survey by A. Ros [17]). Actually, up to now the complete description of minimizers has been achieved only in some special cases; one can see for example $[1,16,19]$ and [21]. However, the study of existence, geometric and topological properties of stationary surfaces (not necessarily minimizers) is far from being complete. Let us mention that Grüter-Jost [4] have proved the existence of minimal discs into convex bodies, while Jost in [6] proved the existence of embedded minimal surfaces of higher genus. In the particular case of the free boundary Plateau problem, some rather global existence results were obtained by M. Struwe in [22,23] and [24]. In [2], the first author proved the existence of surfaces similar to half spheres surrounding a small volume near non-degenerate critical points of the mean curvature of $\partial \Omega$. Here we are interested in the exis- 
tence of families of stationary sets $E_{\varepsilon}$ for the perimeter functional relative to $\Omega$ having small volume meas $E_{\varepsilon}$ proportional to $\varepsilon$. Our result generalizes to higher dimensional sets the one obtained by the first author in [2]. Before stating it some preliminaries are needed. We denote by $\mathcal{V}$ the interior normal vector field along $\partial \Omega$. For a given smooth set $E \subset \Omega$ with finite perimeter, let $\Sigma:=\partial E \cap \Omega$ satisfy $\partial \Sigma \subset \partial \Omega$ and denote by $N$ its exterior normal vector field. For a smooth vector field $\zeta$ in $\mathbb{R}^{m}$, the flow of diffeomorphism $\left\{F_{t}\right\}_{t \in\left(0, t^{*}\right)}$ of $\zeta$ in $\Omega$ induces a variation $\left\{E_{t}=F_{t}(E)\right\}_{t}$ of $E$. Set $A(t)=\mathcal{P}\left(E_{t}, \Omega\right), V(t)=\operatorname{meas}\left(E_{t}\right)$ and

$$
\zeta(p)=\left.\frac{d}{d t} F_{t}(p)\right|_{t=0} .
$$

It is well known that by the first variation of the perimeter and volume functionals, one has

$$
\begin{gathered}
A^{\prime}(0)=-\int_{\Sigma} m H_{\Sigma}\langle\zeta, N\rangle d A+\oint_{\partial \Sigma}\langle\zeta, \bar{N}\rangle d s \\
V^{\prime}(0)=\int_{\Sigma}\langle\zeta, N\rangle d A
\end{gathered}
$$

where $H_{\Sigma}$ is the mean curvature of $\Sigma, N$ its exterior normal vector field and $\bar{N}$ the exterior normal to $\partial \Sigma$ in $\Sigma$. A variation is called normal if $\zeta=\omega N$ for a smooth function $\omega$, admissible if both $F_{t}$ (int $\left.\Sigma\right) \subset \Omega$ and $F_{t}(\partial \Sigma) \subset \partial \Omega$ and volume-preserving if $V(t)=V(0)$ for every $t$. Since for any smooth $\omega$ satisfying $\int_{\Sigma} \omega d A=0$ there exits a volume-preserving admissible normal variation of $E$ with $\zeta=\omega N$, then $E$ is stationary for the perimeter functional $\left(A^{\prime}(0)=0\right)$ for any volume-preserving admissible normal variation of $E$, if and only if

$$
m H_{\Sigma} \equiv \text { const. } \quad \text { in } \quad \Sigma \quad \text { and } \quad \bar{N}(\sigma) \perp T_{\sigma} \partial \Omega \quad \text { for every } \sigma \in \partial \Sigma .
$$

Up to a change of variable, we can reformulate our question to the following free boundary problem: for a given real number $H$, find a hypersurface $\Sigma \subset \Omega_{\varepsilon}$ satisfying the following conditions

$$
\begin{array}{ccc}
H_{\Sigma} & \equiv H \quad \text { in } \Sigma, \\
\partial \Sigma \quad \subset \quad \partial \Omega_{\varepsilon}, \\
\left\langle N, \mathcal{V}^{\varepsilon}\right\rangle=0 \quad \text { on } \partial \Sigma,
\end{array}
$$

where $\Omega_{\varepsilon}:=\varepsilon^{-1} \Omega$ and $\mathcal{V}^{\varepsilon}$ the interior normal vector field on $\partial \Omega_{\varepsilon}$.

If $K$ is a $k$-dimensional smooth submanifold of $\partial \Omega$, we let $n:=m-k$ and define $K_{\varepsilon}:=\varepsilon^{-1} K$. Consider the "half"-geodesic tube contained in $\Omega_{\varepsilon}$ around $K_{\varepsilon}$ of radius 1

$$
\bar{S}_{\varepsilon}\left(K_{\varepsilon}\right):=\left\{q \in \bar{\Omega}_{\varepsilon}: \quad d\left(q, K_{\varepsilon}\right)=1\right\},
$$

with

$$
d\left(q, K_{\varepsilon}\right):=\sqrt{\left|\operatorname{dist}^{\partial \Omega_{\varepsilon}}\left(\tilde{q}, K_{\varepsilon}\right)\right|^{2}+|q-\tilde{q}|^{2}}
$$


where $\tilde{q}$ is the projection of $q$ on $\partial \Omega_{\varepsilon}$ and

$$
\begin{aligned}
& \operatorname{dist}^{\partial \Omega_{\varepsilon}}\left(\tilde{q}, K_{\varepsilon}\right) \\
& =\inf \left\{\operatorname{length}(\gamma): \gamma \in C^{1}([0,1]) \text { is a geodesic in } \partial \Omega_{\varepsilon} ; \gamma(0) \in K_{\varepsilon} ; \gamma(1)=\tilde{q}\right\} .
\end{aligned}
$$

By the smoothness of $\partial \Omega$ and $K$, the tube is a smooth, possibly immersed, hypersurface provided $\varepsilon$ is sufficiently small. This tube by construction meets $\partial \Omega_{\varepsilon}$ perpendicularly. Furthermore the mean curvature of this tube satisfies (see also Paragraph 3.0.5)

$$
m H\left(\bar{S}_{\varepsilon}\left(K_{\varepsilon}\right)\right)=n+\mathcal{O}(\varepsilon)
$$

as $\varepsilon$ tends to zero and hence it is plausible under some rather mild assumptions on $K$ that we might be able to perturb this tube to satisfy (1.3) with $m H \equiv n$. It turns out that this is not known to be possible for every (small) $\varepsilon>0$ but we prove the following theorem :

Theorem 1.1. Let $\Omega$ be a smooth bounded domain of $\mathbb{R}^{m+1}, m \geq 2$. Suppose that $K$ is a non-degenerate minimal submanifold of $\partial \Omega$. Then, there exist a sequence of intervals $I_{i}=\left(\varepsilon_{i}^{-}, \varepsilon_{i}^{+}\right)$, with $\varepsilon_{i}^{-}<\varepsilon_{i}^{+}$and $\lim _{i \rightarrow+\infty} \varepsilon_{i}^{+}=0$ such that, for all $\varepsilon \in$ $I:=\cup_{i} I_{i}$ the "half" geodesic tube $\varepsilon \bar{S}_{\varepsilon}\left(K_{\varepsilon}\right)$ may be perturbed to a hypersurface ${ }_{\varepsilon} S_{\varepsilon}$ satisfying (1.3) with mean curvature $H_{\varepsilon} S_{\varepsilon} \equiv \frac{n}{m} \varepsilon^{-1}$. Namely there exists a family of embedded constant mean curvature hypersurfaces in $\Omega$ with boundary on $\partial \Omega$ and intersecting it perpendicularly.

\section{Remark 1.2.}

- We emphasize that our argument provides also a stationary area separating of $\mathbb{R}^{m+1} \backslash \bar{\Omega}$ when considering the lower hemisphere parameterized by the stereographic projection from the north pole over the unit ball, see Section 3.

- Notice that the surfaces we obtained might have interesting topology. In fact as far as $\varepsilon$ tends to zero, our solutions concentrate along $K$ hence inherit its topological structure. Furthermore we notice that some existence result of various minimal immersions were obtained in [9] and [20].

We believe that the minimality condition on $K$ should also be necessary to obtain a result in spirit of Theorem 1.1, see the last paragraph of [15]. The nondegeneracy condition might fail in some interesting situations, for example when a symmetry is present. In this case however, one can take advantage of it working in a subclass of invariant functions: this might also guarantee existence for all small $\varepsilon$, see [15, Section 5].

- The hypersurface $S_{\varepsilon}$ is a small perturbation of $\bar{S}_{\varepsilon}\left(K_{\varepsilon}\right)$ in the sense that it is the normal graph (for some function whose $L^{\infty}$ norm is bounded by a constant times $\varepsilon$ ) over a small translate of $K_{\varepsilon}$ in $\partial \Omega_{\varepsilon}$ (by some translation whose $L^{\infty}$ norm is bounded by a constant), we refer to Section 4 for the precise formulation of the construction of $S_{\varepsilon}$. 
- This result also remains true for the existence of capillary hypersurfaces in $\Omega$ namely those with stationary area which intersect $\partial \Omega$ in a constant angle $\gamma \in$ $(0, \pi)$ along there boundaries. For more precise comments see Remark 6.3.

To prove the latter theorem, following $[10,15]$ and [25], we parameterize all surfaces nearby $\bar{S}_{\varepsilon}\left(K_{\varepsilon}\right)$ having boundaries in $\partial \Omega_{\varepsilon}$ by two parametric functions $\Phi$ : $K \rightarrow \mathbb{R}^{n}$ and $w: S_{+}^{n} \times \varepsilon^{-1} K \rightarrow \mathbb{R}$. Here

$$
S_{+}^{n}:=\left\{x=\left(x^{1}, \cdots, x^{n+1}\right) \in \mathbb{R}^{n+1}:|x|=1 \text { and } x^{n+1}>0\right\} .
$$

This yields a perturbed tube $S_{\varepsilon}(w, \Phi)$. A standard computations show that the mean curvature $H(w, \Phi)$ of $S_{\varepsilon}(w, \Phi)$ is constant, with the right boundary conditions, is equivalent to solve a system of nonlinear partial differential equations where the principal part is the Jacobi operator about a hypersurface close to $\bar{S}_{\varepsilon}\left(K_{\varepsilon}\right)$. The solvability is based on the invertibility of this linear operator depending on $\varepsilon$ (small parameter). As we will see later, it turns out that this is possible only for some values of $\varepsilon$ tending to zero. Once we have the invertibility our problem becomes readily a fixed point problem that we can solve provided our approximate solution is accurate enough. Our method here is similar in spirit to the one in [10]. It goes back to Malchiodi-Montenegro in [13] (see also [11, 12] and [14], for related issues).

To begin the procedure, we construct first an approximate solution in the following way: let $\left(y^{1}, y^{2} \ldots, y^{k}\right) \in \mathbb{R}^{k}$ (respectively $\left.\left(z^{1}, z^{2} \ldots, z^{n}\right) \in B_{1}^{n}\right)$ be the local coordinate variables on $K_{\varepsilon}$ (respectively on $S_{+}^{n}$ ). Letting $\Phi: K \rightarrow \mathbb{R}^{n}$ and $w: B_{1}^{n} \times K_{\varepsilon} \rightarrow \mathbb{R}$, consider

$$
S_{0}:(y, z) \mapsto y \times \varepsilon^{-1} \Phi(\varepsilon y)+(1+w(y, z)) \Theta(z) .
$$

The surfaces nearby $\bar{S}_{\varepsilon}\left(K_{\varepsilon}\right)$ are parameterized (locally) by

$$
G(y, z):(y, z) \longrightarrow S_{0}(y, z) \longrightarrow F^{\varepsilon}\left(S_{0}(y, z)\right)
$$

where $F^{\varepsilon}: \mathbb{R}^{k} \times \mathbb{R}^{n+1} \rightarrow \bar{\Omega}$ is defined in (2.6) is "an almost isometry" which parameterize a neighborhood of $K_{\varepsilon}$ in $\Omega_{\varepsilon}, B_{1}^{n}$ is the unit ball centered at the origin and $\Theta=\left(\Theta^{1}, \ldots, \Theta^{n}, \Theta^{n+1}\right)$ is the inverse of the stereographic projection from the south pole. Call the image of this map $S_{\varepsilon}(w, \Phi)$, so in particular

$$
S_{\varepsilon}(0,0)=\bar{S}_{\varepsilon}\left(K_{\varepsilon}\right) \text {. }
$$

Notice that since $\left.\Theta^{n+1}\right|_{\partial B_{1}^{n}}=0$, it follows that all these surfaces close to $S_{\varepsilon}\left(K_{\varepsilon}\right)$ parameterized in this way have boundaries on $\partial \Omega_{\varepsilon}$.

One of the main features of this work is that we compute the mean curvature of $S_{\varepsilon}(w, \Phi)$, in Paragraph 3.0.5, which can be done following [10] but in contrast with that paper, we have to gather some new linear and quadratic terms involving $\Phi$ which will be relevant for the solvability. The linearized mean curvature operator about $\bar{S}_{\varepsilon}\left(K_{\varepsilon}\right)$ splits into some linear operators on $w$ and $\Phi$, given by

$$
-\mathcal{L}_{\varepsilon} w-\varepsilon\langle\mathfrak{J} \Phi, \tilde{\Theta}\rangle+\varepsilon \mathcal{L}^{1} w+\varepsilon \mathcal{J}^{1}(\Phi)+\varepsilon^{2} L(w, \Phi),
$$


where $\mathfrak{J}$ is the Jacobi operator about $K$ in the supporting surface $\partial \Omega$, see Subsection 2.2;

$$
\begin{aligned}
\mathcal{L}_{\varepsilon} & :=\varepsilon^{2} \Delta_{K}+\Delta_{S_{+}^{n}}+n \\
\mathcal{J}^{1} \Phi & :=-(3 n+1) \Theta^{n+1} h(\tilde{\Theta})^{a}\left\langle\Phi_{\bar{a}}, \tilde{\Theta}\right\rangle+\Theta^{n+1} h\left(\Phi_{\bar{a}}\right)^{a}+2 \Theta^{n+1} h: \Gamma(\Phi)
\end{aligned}
$$

and $\mathcal{L}^{1}, L(w, \Phi)$ are second order differential operators, see Subsection 2.5, here $h$ (respectively $\Gamma$ ) is the second fundamental form of $\partial \Omega$ (respectively $K$ ) and $h$ : $\Gamma=h_{a b} \Gamma_{a b}$, where summation over repeated indices is understood. The quadratic part of the mean curvature is given by

$$
\begin{aligned}
& \frac{n}{2}\left(\varepsilon w_{\bar{a}}+\left\langle\Phi_{\bar{a}}, \tilde{\Theta}\right\rangle\right)^{2}-\varepsilon\left\langle\Phi_{\bar{a}}, \nabla_{S^{n}} w_{\bar{a}}\right\rangle-2 \varepsilon^{2} \nabla_{K}^{2} w: \Gamma(\Phi) \\
& \quad+\frac{n+2}{6}\langle R(\Phi, \tilde{\Theta}) \Phi, \tilde{\Theta}\rangle-\frac{1}{3}\left\langle R\left(\Phi, E_{i}\right) \Phi, E_{i}\right\rangle+Q(w)+\varepsilon Q(w, \Phi),
\end{aligned}
$$

where $\tilde{\Theta}=\left(\Theta^{1}, \ldots, \Theta^{n}, 0\right)$. Finally the boundary condition reads

$$
\left\langle N, \mathcal{V}^{\varepsilon}\right\rangle=(-1+w) \frac{\partial w}{\partial \eta}+\overline{\mathcal{O}}\left(\varepsilon^{2}\right)+\varepsilon^{2} \bar{L}(w, \Phi)+\varepsilon \bar{Q}(w, \Phi) \quad \text { on } \quad \partial S_{+}^{n} \times K
$$

where $\eta=-E_{n+1}$ is the normal vector field of $\partial S_{+}^{n}$ in $S_{+}^{n}$.

As we will explain later, the Jacobi operator about $\bar{S}_{\varepsilon}\left(K_{\varepsilon}\right)$ (very closed to the operator (1.5)) has inverse norm which blows-up at rate $\frac{1}{\varepsilon^{R}}$ for some $R>0$ and then one do not hope to apply a fixed point argument at this state.

However, we can adjust the tube $\bar{S}_{\varepsilon}\left(K_{\varepsilon}\right)$ as accurate as possible, to a tube $S_{\varepsilon}\left(\hat{w}^{(r)}, \hat{\Phi}^{(r)}\right)$ satisfying (1.7) below. For that, letting $r \geq 1$ be an integer and setting

$$
\hat{w}^{(r)}=\sum_{d=1}^{r} \varepsilon^{d} w^{(d)} \quad \text { and } \quad \hat{\Phi}^{(r)}=\sum_{d=1}^{r-1} \varepsilon^{d} \Phi^{(d)},
$$

we have solved

$$
\begin{aligned}
m H\left(\hat{w}^{(r)}, \hat{\Phi}^{(r)}\right) & =n+\mathcal{O}\left(\varepsilon^{r+1}\right) & \text { in } & S_{\varepsilon}\left(\hat{w}^{(r)}, \hat{\Phi}^{(r)}\right), \\
\left\langle N, \mathcal{V}^{\varepsilon}\right\rangle & =\overline{\mathcal{O}}\left(\varepsilon^{r+2}\right) & \text { on } & \partial S_{\varepsilon}\left(\hat{w}^{(r)}, \hat{\Phi}^{(r)}\right) .
\end{aligned}
$$

This leads to an iterative scheme, see Section 4. The term of order $\mathcal{O}(\varepsilon)$ appearing in the expansion of the mean curvature (Paragraph 3.0.5) depends linearly on the tangential curvature of $K$ which is in the kernel of $\Delta_{S_{+}^{n}}+n$ (spanned by $\Theta^{i}$ with $i=1, \ldots, n)$ and normal curvature $K$ which is perpendicular to this kernel. Consequently by Fredholm theorem, we can kill these terms by $w^{(1)}$ provided $K$ is minimal.

Now to annihilate the higher order terms with suitable couples $\left(w^{(d)}, \Phi^{(d-1)}\right)$, $d \geq 2$, if we project on the kernel of $\Delta_{S_{+}^{n}}+n$, there appears only $\mathfrak{J}$ (the Jacobi 
operator about $K$ ) acting on $\Phi^{(d-1)}$ because when we project, the term $\mathcal{J}^{1} \Phi^{(d-1)}$ disappear by oddness. Moreover neither the nonlinear terms appearing in the expansion of $H(w, \Phi)$ nor the perpendicularity condition will influence the iteration as well. Therefore the non-degeneracy of $K$ is sufficient for this procedure at each step of the iterative scheme. In this way for any integer $r \geq 1$ we will be able to have (1.7) yielding good approximate solutions. We notice that it is more convenient to use the operator $\Delta_{S_{+}^{n}}+n+\langle\mathfrak{J}, \tilde{\Theta}\rangle$ to accomplish this task because it is invertible in $L^{2}\left(S_{+}^{n} \times K\right)$. Unfortunately one cannot use it for full solvability of the problem because $w$ may not gain regularity. We refer to Section 4 for more details.

The final step (see Section 5) is more delicate and consists of the invertibility of the Jacobi operator about $S_{\varepsilon}\left(\hat{w}^{(r)}, \hat{\Phi}^{(r)}\right)$ which we call $\mathbb{L}_{\varepsilon, r}$. Let us mention that at this level all terms in the expansion depend on $r$ except the model operator $-\mathcal{L}_{\varepsilon} w-\varepsilon\langle\mathfrak{J} \Phi, \tilde{\Theta}\rangle$. At first glance one sees that the operator $\mathbb{L}_{\varepsilon, r}$ is not so close to the model one in the usual Sobolev norms because of the competition between the operators $\langle\mathfrak{J} \Phi, \tilde{\Theta}\rangle$ and $\mathcal{L}_{r}^{1}$. This is due to fact that if one consider a tube of radius $\varepsilon$ in a manifold $\mathcal{M}$ with boundary sitting on $\partial \mathcal{M}$, the mean curvature expansion makes appear terms of order $\varepsilon$ depending on the second fundamental form of $\partial \mathcal{M}$. On the contrary, dealing with manifolds without boundary, as in [10], it turns out that in this case the first error terms are of order $\varepsilon^{2}$ and thus also in the expansion of the mean curvature of there perturbed tube, there cannot appear terms like $\varepsilon L$, see [10, Proposition 4.1]. Having bigger error terms than those in [10], we need more accurate approximate solutions and different spaces for the spectral analysis. Since our operator $\mathbb{L}_{\mathcal{E}, r}$ acts on the couple $(w, \Phi)$ almost separately, to tackle this it is natural to adjust the norms used for $w$ and $\Phi$. For any $v \in L^{2}\left(S_{+}^{n} \times K\right)$ we decompose it as $v=\varepsilon^{1-2 s} w+\langle\Phi, \tilde{\Theta}\rangle$ where $\Phi^{i}, i=1, \ldots, n$ are the components of the projection of $v$ onto the Kernel of $\Delta_{S_{+}^{n}}+n$ for some $s \in(0,1 / 2)$. With this decomposition, in a suitable weighted Hilbert subspace of $L^{2}\left(S_{+}^{n} \times K\right)$ we can see $\mathbb{L}_{\varepsilon, r}$ as a perturbation of the model one, see Proposition 5.1.

As mentioned above the existence of families of constant mean curvature surfaces holds only for a suitable sequence of intervals with length decreasing to zero and not the whole $\varepsilon$ is related to a resonance phenomenon peculiar to concentration on positive dimensional sets and it appears in the study of several class of (geometric) non-linear PDE's. Concentration along sets of dimension $k=1, \ldots, n-1$ has been proved here, and analogous spectral properties hold true. By the Weyl's asymptotic formula, if solutions concentrate along a set of dimension $d$ the average distance between those close to zero is of order $\varepsilon^{d}$. The resonance phenomenon was taken care of using a theorem by $\mathrm{T}$. Kato, see [7, page 445], which allows to differentiate eigenvalues with respect to $\varepsilon$. In the aforementioned papers it was shown that, when varying the parameter $\varepsilon$, the spectral gaps near zero almost do not shrink, and invertibility can be obtained for a large family of epsilon's. The case of one dimensional limit sets can be handled using a more direct method based on a Lyapunov-Schmidt reduction, indeed in this case the distance between two consecutive small eigenvalues, candidates to be resonant, is sufficiently large and working away from resonant modes one can perform a contraction mapping ar- 
gument quite easily. Here instead the average distance between two consecutive eigenvalues becomes denser and denser, to overcome this problem one needs to apply Kato's Theorem constructing first good approximate eigenfunctions, we refer to Section 5. And finally following [10], one can estimate the size of the spectral gaps, which determine the size of the norm of the inverse of $\mathbb{L}_{\varepsilon, r}$. For suitable values of $\varepsilon$ the norm of the inverse of $\mathbb{L}_{\varepsilon, r}$ is of order $O\left(\frac{1}{\varepsilon^{R}}\right)$ with a fixed $R>0$ independent of $r$. Now as far as $r$ can be chosen arbitrary large, our fixed point problem can be merely solved. This program is carried out in the last section.

Acknowledgements. The authors wish to thank Professor Andrea Malchiodi for useful discussions and Professor Frank Pacard for pointing out to us the generalization to capillary problems. They are supported by M.U.R.S.T within the PRIN 2006 Variational Methods and Nonlinear Differential Equations and by GNAMPA within the project 2007 Geometric Evolution Equations. F. Mahmoudi is grateful to SISSA for their kind hospitality.

\section{Preliminaries}

Let $K$ be a $k$-dimensional submanifold of $(\partial \Omega, \bar{g})(1 \leq k \leq m-1)$ and set $n=m-$ $k$. We choose along $K$ a local orthonormal frame field $\left(\left(E_{a}\right)_{a=1, \cdots k},\left(E_{i}\right)_{i=1, \cdots, n}\right)$ which is oriented and call $\mathcal{V}$ the interior normal field along $\partial \Omega$ and $\mathcal{V}_{\mid K}=E_{n+1}$. At points of $K, \mathbb{R}^{m+1}$ splits naturally as $T \partial \Omega \oplus \mathbb{R} E_{n+1}$ with $T \partial \Omega=T K \oplus N K$, where $T K$ is the tangent space to $K$ and $N K:=N K^{\partial \Omega}$ represents the normal bundle in $\partial \Omega$, which are spanned respectively by $\left(E_{a}\right)_{a}$ and $\left(E_{j}\right)_{j}$.

\subsection{Fermi coordinates on $\partial \Omega$ near $K$}

Denote by $\nabla$ the connection induced by the metric $\bar{g}$ and by $\nabla^{\perp}$ the corresponding normal connection on the normal bundle. Given $q \in K$, we use some geodesic coordinates $\bar{y}$ centered at $q$.

$$
f: \bar{y} \longrightarrow \exp _{q}^{K}\left(\bar{y}^{a} E_{a}\right)
$$

This yields the coordinate vector fields $\bar{X}_{a}:=f_{*}\left(\partial_{\bar{y}} a\right)$. We also assume that at $q$ the normal vectors $\left(E_{i}\right)_{i}, i=1, \ldots, n$, are transported parallely (with respect to $\nabla^{\perp}$ ) through geodesics from $q$, so in particular

$$
\bar{g}\left(\nabla_{E_{a}} E_{j}, E_{i}\right)=0 \quad \text { at } q, \quad i, j=1, \ldots, n, a=1, \ldots, k .
$$

In a neighborhood of $q$, we choose Fermi coordinates $(\bar{y}, \zeta)$ on $\partial \Omega$ defined by

$$
\bar{F}:(y, \zeta) \longrightarrow \exp _{f(\bar{y})}^{\partial \Omega}\left(\sum_{i=1}^{n} \zeta^{i} E_{i}\right) ; \quad(\bar{y}, \zeta)=\left(\left(\bar{y}^{a}\right)_{a},\left(\zeta^{i}\right)_{i}\right)
$$


Hence we have the coordinate vector fields

$$
\bar{X}_{i}:=\bar{F}_{*}\left(\partial_{\zeta^{i}}\right) \quad \text { and } \quad \bar{X}_{a}:=\bar{F}_{*}\left(\partial_{\bar{y}}{ }^{a}\right) .
$$

By our choice of coordinates, on $K$ the metric $\bar{g}_{\alpha, \beta}:=\left\langle\bar{X}_{\alpha}, \bar{X}_{\beta}\right\rangle$ splits in the following way

$$
\bar{g}(q)=\bar{g}_{a b}(q) d \bar{y}^{a} \otimes d \bar{y}^{b}+\bar{g}_{i j}(q) d \zeta^{i} \otimes d \zeta^{j} ; \quad q \in K .
$$

We denote by $\Gamma_{a}^{b}(\cdot)$ the 1 -forms defined on the normal bundle of $K$ by

$$
\Gamma_{a}^{b}\left(E_{i}\right)=\bar{g}\left(\nabla_{E_{a}} E_{b}, E_{i}\right) .
$$

The submanifold $K$ is said to be minimal if the trace $\Gamma_{a}^{a}(\cdot)=0$.

We will also denote by $R_{\alpha \beta \gamma \delta}$ the components of the curvature tensor with lowered indices, which are obtained by means of the usual ones $R_{\beta \gamma \delta}^{\sigma}$ by

$$
R_{\alpha \beta \gamma \delta}=\bar{g}_{\alpha \sigma} R_{\beta \gamma \delta}^{\sigma} .
$$

When we consider the metric coefficients in a neighborhood of $K$, we obtain a deviation from formula (2.4), which is expressed by the next lemma, see [10, Proposition 2.1] for the proof. Denote by $r$ the distance function from $K$.

Lemma 2.1. In the above coordinates $(\bar{y}, \zeta)$, for any $a=1, \ldots, k$ and any $i, j=$ $1, \ldots, n$, we have

$$
\begin{aligned}
& \bar{g}_{i j}(0, \zeta)=\delta_{i j}+\frac{1}{3} R_{i s t j} \zeta^{s} \zeta^{t}+\mathcal{O}\left(r^{3}\right) \\
& \bar{g}_{a j}(0, \zeta)=\mathcal{O}\left(r^{2}\right) \\
& \bar{g}_{a b}(0, \zeta)=\delta_{a b}-2 \Gamma_{a}^{b}\left(E_{i}\right) \zeta^{i}+\left[R_{s a b l}+\Gamma_{a}^{c}\left(E_{s}\right) \Gamma_{c}^{b}\left(E_{l}\right)\right] \zeta^{s} \zeta^{l}+\mathcal{O}\left(r^{3}\right)
\end{aligned}
$$

Here $R_{i s t j}$ are computed at the point $q$ of $K$ parameterized by $(0,0)$.

The boundary of the scaled domain $\partial \Omega_{\varepsilon}:=\frac{1}{\varepsilon} \partial \Omega$ is parameterized, in a neighborhood of $\varepsilon^{-1} q \in K_{\varepsilon}:=\varepsilon^{-1} K$ by

$$
\bar{F}^{\varepsilon}\left(y, x^{\prime}\right):=\frac{1}{\varepsilon} \bar{F}\left(\varepsilon y, \varepsilon x^{\prime}\right) \quad \text { with } x^{\prime}:=\left(x^{i}, \cdots, x^{n}\right) .
$$

Hence we have the induced coordinate vector fields

$$
X_{i}:=\bar{F}_{*}^{\varepsilon}\left(\partial_{x^{i}}\right) \quad \text { and } \quad X_{a}:=\bar{F}_{*}^{\varepsilon}\left(\partial_{y^{a}}\right) .
$$

By construction, $X_{\alpha \mid \varepsilon^{-1} q}=E_{\alpha}$ and $\mathcal{V}^{\varepsilon}\left(\varepsilon^{-1} q\right)=E_{n+1}$. From Lemma 2.1 it is evident that the metric $g$ on $\left(\partial \Omega_{\varepsilon}, g\right)$ has the expansion given by the 
Lemma 2.2. In a neighborhood of $K_{\varepsilon}$ the following hold

$$
\begin{aligned}
& g_{i j}(0, x)=\delta_{i j}+\frac{\varepsilon}{3} R_{i s t j} x^{s} x^{t}+\mathcal{O}\left(\varepsilon^{2} r^{3}\right) ; \\
& g_{a j}(0, x)=\mathcal{O}\left(\varepsilon r^{2}\right) ; \\
& g_{a b}(0, x)=\delta_{a b}-2 \Gamma_{a}^{b}\left(E_{i}\right) x^{i}+\varepsilon\left[R_{s a b l}+\Gamma_{a}^{c}\left(E_{s}\right) \Gamma_{c}^{b}\left(E_{l}\right)\right] x^{s} x^{l}+\mathcal{O}\left(\varepsilon^{2} r^{3}\right) .
\end{aligned}
$$

We can now parameterize tubular neighborhood of $K_{\varepsilon}$ in $\Omega_{\varepsilon}$,

$$
F^{\varepsilon}\left(y, x^{\prime}, x^{n+1}\right)=\frac{1}{\varepsilon} \bar{F}\left(\varepsilon y, \varepsilon x^{\prime}\right)+x^{n+1} \mathcal{V}^{\varepsilon}\left(y, x^{\prime}\right),
$$

where $\mathcal{V}^{\varepsilon}\left(y, x^{\prime}\right):=\mathcal{V}\left(\frac{1}{\varepsilon} \bar{F}\left(\varepsilon y, \varepsilon x^{\prime}\right)\right)$. We denote by $h$ the second fundamental form of $\partial \Omega$ so that:

$$
\left\langle d \mathcal{V}^{\varepsilon}(p)\left[X_{\alpha}\right], X_{\beta}\right\rangle=\varepsilon h_{\alpha, \beta}(q)
$$

when $q=\bar{F}^{\varepsilon}(p)$.

\subsection{The Jacobi operator about $K$}

The linearized mean curvature operator about $K$ is given by

$$
\mathfrak{J}:=\Delta^{\perp}-\mathcal{R}^{\perp}+\mathcal{B}
$$

where the normal Laplacian $\Delta^{\perp}$ is defined as

$$
\Delta^{\perp}:=\nabla_{E_{a}}^{\perp} \nabla_{E_{a}}^{\perp}-\nabla_{\nabla_{a}}^{\perp} E_{a},
$$

with $\nabla^{\perp}$ denoting the connection on the normal bundle of $K$ in $\partial \Omega$ while $\mathcal{B}$ is a symmetric operator defined by

$$
\bar{g}(\mathcal{B}(X), Y)=\Gamma_{a}^{b}(X) \Gamma_{b}^{a}(Y) \quad \text { for all } X, Y \in N K,
$$

where $\Gamma$ is defined in (2.5) and $\mathcal{R}^{\perp}: N_{p} K \longrightarrow N_{p} K$ is given by

$$
\mathcal{R}^{\perp}:=\left(R\left(E_{a}, \cdot\right) E_{a}\right)^{\perp},
$$

where $(\cdot)^{\perp}$ denotes the orthogonal projection on $N K$. The Ricci tensor is defined by

$$
\operatorname{Ric}(X, Y)=-\bar{g}\left(R\left(X, E_{\gamma}\right) Y, E_{\gamma}\right) \quad \text { for all } X, Y \in T_{p} M .
$$

Finally, we recall that the submanifold $K$ is said to be non-degenerate if the Jacobi operator $\mathfrak{J}$ is invertible, or equivalently if the equation $\mathfrak{J} \Phi=0$ has only the trivial solution among the sections in $N K$. 


\subsection{First and second variation of area for capillary hypersurfaces}

Let $\Sigma$ be a smooth hypersurface in an $(m+1)$-dimensional Riemannian manifold $(M, g)$ with smooth, nonempty boundary $\partial M$. Suppose that $\partial \Sigma \subset \partial M$ so that $M$ is separated into two parts, call $\Lambda$ the boundary of one of these parts in $\partial M$.

\subsubsection{First variation of area}

Let $F_{t}$ be a variation of $\Sigma$ with variation vector field

$$
\zeta(p)=\frac{\partial F_{t}}{\partial t}(p)_{\mid t=0} \quad \text { for every } p \in \Sigma .
$$

A variation is called admissible if both $F_{t}(\operatorname{int} \Sigma) \subset M$ and $F_{t}(\partial \Sigma) \subset \partial M$. Let $N$ be a unit outer normal vector along $\Sigma ; H_{\Sigma}$ its mean curvature and $v$ (respectively $\bar{v}$ ) be the unit exterior normal vector along $\partial \Sigma$ in $\Sigma$ (respectively in $\Lambda$ ).

An admissible variation induces hypersurfaces $\Sigma_{t}$ and $\Lambda_{t}$. Let $A(t)$ (respectively $T(t)$ ) be the volume of $\Sigma_{t}$ (respectively $\Lambda_{t}$ ) and $V(t)$ the signed volume bounded by $\Sigma$ and $\Sigma_{t}$. For a given angle $\gamma \in(0, \pi)$, we consider the total energy

$$
\mathcal{E}(t):=A(t)-\cos (\gamma) T(t) .
$$

It is well known (see for example [18]) that

$$
\mathcal{E}^{\prime}(0)=-\int_{\Sigma} n H_{\Sigma}\langle\zeta, N\rangle_{g} d A+\oint_{\partial \Sigma}\langle\zeta, v-\cos (\gamma) \bar{v}\rangle_{g} d s
$$

and

$$
V^{\prime}(0)=\int_{\Sigma}\langle\zeta, N\rangle_{g} d A
$$

A variation is called volume-preserving if $V(t)=V(0)$ for every $t . \Sigma$ is called capillary hypersurface if $\Sigma$ is stationary for the total energy $\left(\mathcal{E}^{\prime}(0)=0\right)$ for any volume-preserving admissible variation. Consequently if $\Sigma$ is capillary, it has a constant mean curvature and intersect $\partial M$ with the angle $\gamma$ in the sense that the angle between the normals of $v$ and $\bar{v}$ is $\gamma$ or equivalently the angle between $N$ and $\mathcal{V}$ is $\gamma$, where $\mathcal{V}$ is the unit outer normal field along $\partial M$.

Physically, in the tree-phase system the quantity $\cos (\gamma) T(0)$ is interpreted as the wetting energy and $\gamma$ the contact angle while $\cos (\gamma)$ is the relative adhesion coefficient between the fluid bounded by $\Sigma$ and $\Lambda$ and the walls $\partial M$. Here we are interested in a configuration in the absence of gravity. A more general setting including the gravitational energy and works on capillary surfaces can be found in the book by R. Finn [3].

\subsubsection{The Jacobi operator about $\Sigma$}

We denote by $\Pi_{\Sigma}$ and $\Pi_{\partial M}$ the second fundamental forms of $\Sigma$ and $\partial M$ respectively. Assume that $\Sigma$ is a capillary hypersurface. Recall that the Jacobi operator (the linearized mean curvature operator about $\Sigma$ ) is given by the second variation 
of the total energy functional $\mathcal{E}$. For any volume-preserving admissible variation, we have (see for the proof [18, Appendix])

$$
\begin{aligned}
\mathcal{E}^{\prime \prime}(0)= & -\int_{\Sigma}\left(\omega \Delta_{\Sigma} \omega+\left|\Pi_{\Sigma}\right|^{2} \omega^{2}+\operatorname{Ric}_{g}(N, N) \omega^{2}\right) d A \\
& +\oint_{\partial \Sigma}\left(\omega \frac{\partial \omega}{\partial v}-q \omega^{2}\right) d s
\end{aligned}
$$

where

$$
\omega=\langle\zeta, N\rangle_{g} \quad \text { and } \quad q=\frac{1}{\sin (\gamma)} \Pi_{\partial M}(\bar{v}, \bar{v})-\cot (\gamma) \Pi_{\Sigma}(v, v) .
$$

Since for any smooth $\omega$ with $\int_{\Sigma} \omega d A=0$ there exits an admissible, volumepreserving variation with variation vector field $\omega N$ as a normal part, we have now the Jacobi operator about $\Sigma$ that we define by duality as

$$
\left\langle\mathfrak{L}_{\Sigma, N} \omega, \omega^{\prime}\right\rangle:=\int_{\Sigma}\left\{\nabla \omega \nabla \omega^{\prime}-\left(\left|\Pi_{\Sigma}\right|^{2}+\operatorname{Ric}_{g}(N, N)\right) \omega \omega^{\prime}\right\} d A+\oint_{\partial \Sigma} q \omega \omega^{\prime} d s .
$$

Remark 2.3. Let us observe that any smooth transverse vector field $\hat{N}$ along $\Sigma$ induces admissible volume preserving variation. The linearized mean curvature operators $\mathfrak{L}_{\Sigma, N}$ and $\mathfrak{L}_{\Sigma, \hat{N}}$ are linked by

$$
\mathfrak{L}_{\Sigma, \hat{N}} \hat{\omega}=\mathfrak{L}_{\Sigma, N}\left(\langle N, \hat{N}\rangle_{g} \hat{\omega}\right)+m \hat{N}^{T}\left(H_{\Sigma}\right) \hat{\omega}
$$

where $\hat{N}^{T}$ is the orthogonal projection of $\hat{N}$ on $T \Sigma$. This shows that $\mathfrak{L}_{\Sigma, \hat{N}}$ is selfadjoint with respect to the inner product

$$
\int_{\Sigma} \hat{\omega} \hat{\omega}^{\prime}\langle N, \hat{N}\rangle_{g} d A
$$

\subsection{The stereographic projection}

We will denote by $\mathbf{p}: \mathbb{R}^{n} \rightarrow S^{n}$ the inverse of the stereographic projection from the south pole. $\mathbf{p}=\left(\mathbf{p}^{1}, \ldots, \mathbf{p}^{n}, \mathbf{p}^{n+1}\right)$ is a conformal parametrization of $S^{n}$ and for any $z=\left(z^{1}, \ldots, z^{n}\right) \in \mathbb{R}^{n}$,

$$
\begin{aligned}
\mathbf{p}(z) & =(z, 1) \mu(z)-E_{n+1} \\
& =\left(\frac{2 z^{1}}{1+|z|^{2}}, \ldots, \frac{2 z^{n}}{1+|z|^{2}}, \frac{1-|z|^{2}}{1+|z|^{2}}\right)
\end{aligned}
$$


with conformal factor given by

$$
\mu(z):=\frac{2}{1+|z|^{2}} .
$$

We often use the projection of $\mathbf{p}$ on $\mathbb{R}^{n}$ and denote it by

$$
\tilde{\mathbf{p}}(z):=(z, 0) \mu(z) .
$$

We collect in the following lemma some properties of the function $\mathbf{p}$ which will be useful later on, we omit here the proof which can be obtained rather easily with elementary computations

Lemma 2.4. For every $i, j, l=1, \ldots, n$, there holds

$$
\begin{gathered}
\left\langle\mathbf{p}_{i}, \mathbf{p}_{j}\right\rangle=\mu^{2} \delta_{i j} ; \quad \mathbf{p}_{i}^{n+1}=-\mu \mathbf{p}^{i} ; \quad \tilde{\mathbf{p}}_{i}=-\mathbf{p}^{i} \tilde{\mathbf{p}}+\mu E_{i} ; \\
\left\langle\mathbf{p}_{i i}, \mathbf{p}_{l}\right\rangle=\mu^{2} \mathbf{p}^{l}-2 \mu^{2} \mathbf{p}^{i} \delta_{i l} .
\end{gathered}
$$

Here $\mathbf{p}_{i}$ and $\mathbf{p}_{i j}$ stands for $\frac{\partial \mathbf{p}}{\partial z^{i}}$ and $\frac{\partial^{2} \mathbf{p}}{\partial z^{i} \partial z^{j}}$ respectively.

Recall that the Laplace operator on $S^{n}$ can be expressed in terms of the Euclidean one by the formula

$$
\Delta_{S^{n}}=\frac{1}{\mu^{2}}\left(\Delta_{\mathbb{R}^{n}}-\left\langle\mathbf{p}_{i i}, \mathbf{p}_{k}\right\rangle \partial_{k}\right) .
$$

Moreover, it is easy to verify that

$$
\Delta_{S^{n}} \mathbf{p}+n \mathbf{p}=0 .
$$

It is clear that for any $0<r \leq 1$ the restriction of $\mathbf{p}$ on $B_{r}^{n}$ parameterizes a spherical cap $S^{n}(r)$, where $B_{r}^{n}$ is the ball centered at 0 with radius $r$.

Given $\gamma \in(0, \pi)$, if we let $r^{2}=\frac{1-\cos (\gamma)}{1+\cos (\gamma)}$, the image by $\mathbf{p}$ of $B_{r}^{n}$ is the spherical cap $S^{n}(\gamma)$ which intersects the horizontal plane $\mathbb{R}^{n}+\cos (\gamma) E_{n+1}$ and makes an angle $\gamma$ with it. In particular we denote (henceforth define)

$$
\begin{gathered}
\Theta(\gamma):=\left.\mathbf{p}\right|_{B_{r(\gamma)}^{n}}-\cos (\gamma) E_{n+1} ; \quad \Theta:=\Theta\left(\frac{\pi}{2}\right) \\
S_{+}^{n}:=S^{n}\left(\frac{\pi}{2}\right)=\left\{x=\left(x^{1}, \ldots, x^{n+1}\right) \in \mathbb{R}^{n+1} \quad: \quad|x|=1 \text { and } x^{n+1}>0\right\} .
\end{gathered}
$$

For any $0<r \leq 1$, denote by $\tau_{r}$ the unit outer normal vector of $\partial B_{r}^{n}$, the normal field (not unitary) of $\partial S^{n}(r)$ in $S^{n}(r)$ expressed as follows

$$
\left.\frac{\partial \mathbf{p}}{\partial \tau_{r}}\right|_{\partial B_{r}^{n}}=\left.\mu|\tilde{\mathbf{p}}|\left(\mathbf{p}^{n+1} \frac{\tilde{\mathbf{p}}}{|\tilde{\mathbf{p}}|^{2}}-E_{n+1}\right)\right|_{\partial B_{r}^{n}} .
$$


Now when $r^{2}=\frac{1-\cos (\gamma)}{1+\cos (\gamma)}$, the unit normal in $S^{n}(\gamma)$ of $\partial S^{n}(\gamma)$ is given and denoted by

$$
\eta(\gamma)=\cot (\gamma) \tilde{\Theta}(\gamma)-\sin (\gamma) E_{n+1} \text {, in particular } \eta:=\eta\left(\frac{\pi}{2}\right)=-E_{n+1}
$$

while the unit normal of $\partial S^{n}(\gamma)$ in the plane $\mathbb{R}^{n}+\cos (\gamma) E_{n+1}$ is $\frac{\tilde{\Theta}(\gamma)}{|\tilde{\Theta}(\gamma)|} \mid \partial B_{r}^{n}$.

Observe that the angle between the two normals $\frac{\tilde{\Theta}(\gamma)}{|\tilde{\Theta}(\gamma)|}$ and $\eta(\gamma)$ is $\gamma$ along $\partial S^{n}(\gamma)$, namely since $|\tilde{\Theta}(\gamma)|=\sin (\gamma)$ on $\partial B_{r}^{n}$,

$$
\left\langle\frac{\tilde{\Theta}(\gamma)}{|\tilde{\Theta}(\gamma)|}, \eta(\gamma)\right\rangle=\cos (\gamma) \quad \text { on } \partial S^{n}(\gamma) .
$$

Consider the eigenvalue problem, $u: S^{n}(\gamma) \rightarrow \mathbb{R}$,

$$
\begin{cases}\Delta_{S^{n}(\gamma)} u+n u=0 & \text { in } S^{n}(\gamma) \\ \frac{\partial u}{\partial \eta(\gamma)}=\cot (\gamma) u & \text { on } \partial S^{n}(\gamma) .\end{cases}
$$

It is well known that the only solutions to the interior equation are the degree one homogeneous polynomials on $S_{+}^{n}$, spanned by the $n+1$ components of $\mathbf{p}$. By (2.15) the boundary condition is satisfied only by $\Theta^{i}(\gamma), i=1, \cdots, n$.

\subsection{Notation}

In the following, expressions of the form $L(w, \Phi)$ denote linear operators, in the functions $w$ and $\Phi^{j}$ as well as their derivatives with respect to the vector fields $\varepsilon X_{a}$ and $X_{i}$ up to second order, the coefficients of which are smooth functions on $S^{n}(\gamma) \times K$ bounded by a constant independent of $\varepsilon$ in the $\mathcal{C}^{\infty}$ topology (where derivatives are taken using the vector fields $X_{\bar{a}}$ and $\left.X_{i}\right)$. Also $\bar{L}(w, \Phi)$ are restrictions of expressions like $L(w, \Phi)$ on $\partial S^{n}(\gamma) \times K$ with $L(w, \Phi)$ contains only one derivative of $w$ or $\Phi$ with respect to the vector fields $\varepsilon X_{a}$ and $X_{i}$.

Similarly, expressions of the form $Q(w, \Phi)$ denote nonlinear operators, in the functions $w$ and $\Phi^{j}$ as well as their derivatives with respect to the vector fields $\varepsilon X_{a}$ and $X_{i}$ still up to second order, whose coefficients of the Taylor expansion are smooth functions on $S^{n}(\gamma) \times K$ which are bounded by a constant independent of $\varepsilon$ in $\mathcal{C}^{\infty}$ topology (where derivatives are taken using the vector fields $X_{a}$ and $X_{i}$ ). Moreover, $Q$ vanish quadratically in the pair $(w, \Phi)$ at 0 (that is, its Taylor expansion does not involve any constant nor any linear term). Also $\bar{Q}(w, \Phi)$ are restrictions of expressions like $Q(w, \Phi)$ on $\partial S^{n}(\gamma) \times K$ with $Q(w, \Phi)$ contains only one derivative of $w$ or $\Phi$ with respect to the vector fields $\varepsilon X_{a}$ and $X_{i}$.

Finally, terms denoted $\mathcal{O}\left(\varepsilon^{d}\right)$ are smooth functions on $S^{n}(\gamma) \times K_{\varepsilon}$ which are bounded by a constant times $\varepsilon^{d}$ in $\mathcal{C}^{\infty}$ topology (where derivatives are taken using the vector fields $X_{a}$ and $\left.X_{i}\right)$. Also expressions like $\overline{\mathcal{O}}\left(\varepsilon^{d}\right)$ are restrictions of $\mathcal{O}\left(\varepsilon^{d}\right)$ on $\partial S^{n}(\gamma) \times K$. 


\section{Geometry of tubes}

We derive expansions as $\varepsilon$ tends to 0 for the metric, second fundamental form and mean curvature of $\bar{S}_{\varepsilon}\left(K_{\varepsilon}\right)$ and their perturbations.

\subsubsection{Perturbed tubes}

We now describe a suitable class of deformations of the geodesic tubes (in the metric induced by $F^{\varepsilon}$ on $\left.\mathbb{R}^{m+1}\right) \bar{S}_{\varepsilon}\left(K_{\varepsilon}\right)$, depending on a section $\Phi$ of $N K_{\varepsilon}:=$ $S_{+}^{n} \times K_{\varepsilon}$ and a scalar function $w$ on the spherical normal bundle $\left(S N K_{\varepsilon}\right)_{+}$in $\partial \Omega_{\varepsilon}$. We recall that $\left(y^{1}, y^{2} \ldots, y^{k}\right) \in \mathbb{R}^{k}$ (respectively $\left(z^{1}, z^{2} \ldots, z^{n}\right) \in B_{1}^{n}$ ) are the local coordinate variables on $K_{\varepsilon}$ (respectively on $S_{+}^{n}$ ). Letting $\Phi: K \rightarrow \mathbb{R}^{n}$ and $w: B_{1}^{n} \times K_{\varepsilon} \rightarrow \mathbb{R}$, consider

$$
S_{0}:(y, z) \mapsto y \times \varepsilon^{-1} \Phi(\varepsilon y)+(1+w(y, z)) \Theta(z) .
$$

The nearby surfaces of $\bar{S}_{\varepsilon}\left(K_{\varepsilon}\right)$ is parameterized (locally) by

$$
G(y, z):(y, z) \longrightarrow S_{0}(y, z) \longrightarrow F^{\varepsilon}\left(S_{0}(y, z)\right)
$$

namely

$$
G(y, z):=F^{\varepsilon}\left(y, \frac{1}{\varepsilon} \Phi(\varepsilon y)+(1+w(y, z)) \tilde{\Theta}(z),(1+w(y, z)) \Theta^{n+1}(z)\right) .
$$

Since $\left.\Theta^{n+1}\right|_{\partial B_{1}^{n}}=0$, it follows

$$
\left.G(y, z)\right|_{\partial B_{1}^{n}} \in \partial \Omega_{\varepsilon} \quad \text { for any } y .
$$

The image of this map will be called $S_{\varepsilon}(w, \Phi)$. In particular

$$
S_{\varepsilon}(0,0)=\bar{S}_{\varepsilon}\left(K_{\varepsilon}\right) .
$$

It will be understood that for any fixed point $p=F^{\varepsilon}(y, 0) \in K_{\varepsilon}, \Phi(\varepsilon y) \in N K_{\varepsilon} \subset$ $T_{p} \partial \Omega_{\varepsilon}$ and $\Theta(z) \in S_{+}^{n} \subset N K_{\varepsilon} \oplus \mathbb{R} E_{n+1}$ are in the tangent space at $p$ of $\mathbb{R}^{m+1}$ endowed with the metric induced by $F^{\varepsilon}$. For more convenience we introduce the following:

Notation. On $K_{\varepsilon}$ we will consider

$$
\begin{gathered}
\Phi:=\Phi^{j} E_{j} \quad \Phi_{a}:=\partial_{y^{a}} \Phi^{j} E_{j} \quad \Phi_{a b}:=\partial_{y^{a}} \partial_{y^{b}} \Phi^{j} E_{j} \\
\Theta:=\Theta^{j} E_{j}+\Theta^{n+1} E_{n+1}=\tilde{\Theta}+\Theta^{n+1} E_{n+1} \\
\Theta_{i}:=\partial_{z^{i}} \Theta^{j} E_{j}+\partial_{z^{i}} \Theta^{n+1} E_{n+1}=\tilde{\Theta}_{i}+\Theta_{i}^{n+1} E_{n+1} .
\end{gathered}
$$


For simplicity, we will write

$$
\begin{aligned}
w_{j} & :=\partial_{z^{j}} w \\
w_{a} & :=\partial_{y^{a}} w \\
w_{i j} & :=\partial_{z^{i}} \partial_{z^{j}} w ; \\
w_{a b} & :=\partial_{y^{a}} \partial_{y^{b}} w \\
w_{a j} & :=\partial_{y^{a}} \partial_{z^{j}} w .
\end{aligned}
$$

It is easy to see that the tangent space to $S_{\varepsilon}(w, \Phi)$ is spanned by the vector fields

$$
\begin{aligned}
& Z_{a}=G_{*}\left(\partial_{y^{a}}\right)=X_{a}+w_{a} \Upsilon+\Psi_{a}+(1+w) \Theta^{n+1} D_{a} \mathcal{V}^{\varepsilon}, \quad a=1, \ldots, k \\
& Z_{j}=G_{*}\left(\partial_{z^{j}}\right)=(1+w) \Upsilon_{j}+w_{j} \Upsilon+(1+w) \Theta^{n+1} D_{j} \mathcal{V}^{\varepsilon}, \quad j=1, \ldots, n,
\end{aligned}
$$

where

$$
\begin{array}{ll}
\Psi:=\Phi^{j} X_{j} ; & \Psi_{a}:=\partial_{y^{a}} \Phi^{j} X_{j} \\
\Upsilon:=\Theta^{j} X_{j}+\Theta^{n+1} \mathcal{V}^{\varepsilon} ; & \Upsilon_{i}:=\partial_{z^{i}} \Theta^{j} X_{j}+\partial_{z^{i}} \Theta^{n+1} \mathcal{V}^{\varepsilon}
\end{array}
$$

and

$$
\begin{aligned}
& D_{a} \mathcal{V}^{\varepsilon}\left(y,(1+w(y, z)) \tilde{\Theta}+\varepsilon^{-1} \Phi(\varepsilon y)\right)=\varepsilon\left(h_{a \alpha}+\left(w_{a} \Theta^{l}+\Phi_{a}^{l}\right) h_{l \alpha}\right) X_{\alpha} \\
& D_{j} \mathcal{V}^{\varepsilon}\left(y,(1+w(y, z)) \tilde{\Theta}+\varepsilon^{-1} \Phi(\varepsilon y)\right)=\varepsilon\left(w_{j} \Theta^{l}+(1+w) \Theta_{j}^{l}\right) h_{l \alpha} X_{\alpha} .
\end{aligned}
$$

\subsubsection{The first fundamental form}

In this paragraph we expand the coefficients of the first fundamental form of $S_{\varepsilon}(w, \Phi)$. Using the expansions in Lemma 2.2, one can easily get

$$
\begin{aligned}
\left\langle X_{a}, X_{b}\right\rangle= & \delta_{a b}-2 \varepsilon \Gamma_{a}^{b}(\Theta)-2 \Gamma_{a}^{b}(\Phi)+\mathcal{O}\left(\varepsilon^{2}\right)+\varepsilon L(w, \Phi)+Q(w, \Phi) \\
\left\langle X_{i}, X_{j}\right\rangle= & \delta_{i j}+\frac{\varepsilon}{3}\left(\left\langle R\left(\Theta, E_{i}\right) \Phi, E_{j}\right\rangle+\left\langle R\left(\Phi, E_{i}\right) \Theta, E_{j}\right\rangle\right) \\
& +\frac{1}{3}\left\langle R\left(\Phi, E_{i}\right) \Phi, E_{j}\right\rangle+\mathcal{O}\left(\varepsilon^{2}\right)+\varepsilon^{2} L(w, \Phi)+\varepsilon Q(w, \Phi) \\
\left\langle X_{i}, X_{a}\right\rangle= & \mathcal{O}\left(\varepsilon^{2}\right)+\varepsilon L(w, \Phi)+Q(w, \Phi)
\end{aligned}
$$

These together with the fact that $R(\tilde{\Theta}, \tilde{\Theta})=0$ imply

$$
\begin{aligned}
\left\langle\Upsilon, \Upsilon_{j}\right\rangle= & \frac{\varepsilon}{3}\left\langle R(\Phi, \tilde{\Theta}) \tilde{\Theta}, \tilde{\Theta}_{j}\right\rangle+\frac{1}{3}\left\langle R(\Phi, \tilde{\Theta}) \Phi, \tilde{\Theta}_{j}\right\rangle \\
& +\mathcal{O}\left(\varepsilon^{2}\right)+\varepsilon^{2} L(w, \Phi)+\varepsilon Q(w, \Phi) .
\end{aligned}
$$


Using similar arguments, and the fact that $\langle\Upsilon, \Upsilon\rangle=1$ on $K_{\varepsilon}$ yields

$$
\langle\Upsilon, \Upsilon\rangle=1+\frac{1}{3}\langle R(\Phi, \tilde{\Theta}) \Phi, \tilde{\Theta}\rangle+\mathcal{O}\left(\varepsilon^{2}\right)+\varepsilon^{2} L(w, \Phi)+Q(w, \Phi)
$$

Moreover

$$
\begin{aligned}
\left\langle\Upsilon_{i}, \Upsilon_{j}\right\rangle= & \left\langle\Theta_{i}, \Theta_{j}\right\rangle+\frac{1}{3}\left(\left\langle R\left(\Phi, \tilde{\Theta}_{i}\right) \tilde{\Theta}, \tilde{\Theta}_{j}\right\rangle+\left\langle R\left(\Phi, \tilde{\Theta}_{j}\right) \tilde{\Theta}_{,} \tilde{\Theta}_{i}\right\rangle\right) \\
& +\frac{1}{3}\left\langle R\left(\Phi, \tilde{\Theta}_{i}\right) \Phi, \tilde{\Theta}_{j}\right\rangle+\mathcal{O}\left(\varepsilon^{2}\right)+\varepsilon^{2} L(w, \Phi)+Q(w, \Phi) .
\end{aligned}
$$

Now, by (3.2) we have that

$$
\begin{aligned}
\left\langle D_{j} \mathcal{V}^{\varepsilon}, \Upsilon\right\rangle= & \varepsilon(1+w)\left\langle h(\tilde{\Theta}), \tilde{\Theta}_{j}\right\rangle+\varepsilon w_{j}\langle h(\tilde{\Theta}), \tilde{\Theta}\rangle \\
& +\mathcal{O}\left(\varepsilon^{2}\right)+\varepsilon^{2} L(w, \Phi)+\varepsilon Q(w, \Phi)
\end{aligned}
$$

and

$$
\begin{aligned}
\left\langle D_{j} \mathcal{V}^{\varepsilon}, \Upsilon_{i}\right\rangle= & \varepsilon(1+w)\left\langle h\left(\tilde{\Theta}_{i}\right), \tilde{\Theta}_{j}\right\rangle+\varepsilon w_{j}\left\langle h(\tilde{\Theta}), \tilde{\Theta}_{i}\right\rangle \\
& +\mathcal{O}\left(\varepsilon^{2}\right)+\varepsilon^{2} L(w, \Phi)+\varepsilon Q(w, \Phi) .
\end{aligned}
$$

We are now in a position to expand the coefficients of the first fundamental form of $S_{\varepsilon}(w, \Phi)$. We have:

Proposition 3.1. For any $a, b \in\{1, \cdots, k\}$ and $i, j \in\{1, \cdots, n\}$, we have that

$$
\begin{aligned}
\left\langle Z_{a}, Z_{b}\right\rangle= & \delta_{a b}+2 \varepsilon \Theta^{n+1} h_{a b}-2 \varepsilon \Gamma_{a}^{b}(\tilde{\Theta})-2 \Gamma_{a}^{b}(\Phi)+\mathcal{O}\left(\varepsilon^{2}\right) \\
& +\varepsilon L(w, \Phi)+Q(w, \Phi) \\
\left\langle Z_{a}, Z_{j}\right\rangle= & 2 \varepsilon \Theta^{n+1} h\left(\tilde{\Theta}_{j}\right)^{a}+\left\langle\Phi_{\bar{a}}, \tilde{\Theta}_{j}\right\rangle+\mathcal{O}\left(\varepsilon^{2}\right)+\varepsilon L(w, \Phi)+Q(w, \Phi) \\
\left\langle Z_{i}, Z_{j}\right\rangle= & \left\langle\Theta_{i}, \Theta_{j}\right\rangle(1+2 w)+2 \varepsilon(1+3 w) \Theta^{n+1}\left\langle h\left(\tilde{\Theta}_{i}\right), \tilde{\Theta}_{j}\right\rangle \\
& +2 \varepsilon \Theta^{n+1}\left(\left\langle h\left(\tilde{\Theta}_{i}\right), \tilde{\Theta}\right\rangle w_{j}+\left\langle h\left(\tilde{\Theta}_{j}\right), \tilde{\Theta}\right\rangle w_{i}\right) \\
& +\frac{\varepsilon}{3}\left(\left\langle R\left(\tilde{\Theta}, \tilde{\Theta}_{i}\right) \Phi, \tilde{\Theta}{ }_{j}\right\rangle+\left\langle R\left(\tilde{\Theta}, \tilde{\Theta}_{j}\right) \Phi, \tilde{\Theta}_{i}\right\rangle\right)+w_{i} w_{j} \\
& +\left\langle\Theta_{i}, \Theta j\right\rangle w^{2}+\frac{1}{3}\left\langle R\left(\Phi, \tilde{\Theta}_{i}\right) \Phi, \tilde{\Theta}_{j}\right\rangle+\mathcal{O}\left(\varepsilon^{2}\right) \\
& +\varepsilon^{2} L(w, \Phi)+\varepsilon Q(w, \Phi) .
\end{aligned}
$$

\subsubsection{The normal vector field}

In this paragraph we expand the unit normal to $S_{\varepsilon}(w, \Phi)$. Define the vector field

$$
\tilde{N}:=-\Upsilon+\alpha^{j} Z_{j}+\beta^{c} Z_{c}
$$


it is the outer normal field along $S_{\varepsilon}(w, \Phi)$ if we can determine $\alpha^{j}$ and $\beta^{c}$ so that $\tilde{N}$ is orthogonal to all of the $Z_{b}$ and $Z_{i}$. This leads to a linear system for $\alpha^{j}$ and $\beta^{a}$.

We have the following expansions

$$
\begin{aligned}
\left\langle\Upsilon, Z_{a}\right\rangle= & w_{a}+\left\langle\Phi_{\bar{a}}, \tilde{\Theta}\right\rangle+\varepsilon \Theta^{n+1}(h(\tilde{\Theta}))^{a}+\varepsilon^{2} L(w, \Phi)+\varepsilon Q(w, \Phi) ; \\
\left\langle\Upsilon, Z_{j}\right\rangle= & w_{j}+\varepsilon(1+2 w) \Theta^{n+1}\left\langle h(\tilde{\Theta}), \tilde{\Theta}_{j}\right\rangle+2 \varepsilon \Theta^{n+1} w_{j}\langle h(\tilde{\Theta}), \tilde{\Theta}\rangle \\
& +\frac{\varepsilon}{3}\left\langle R(\Phi, \tilde{\Theta}) \tilde{\Theta}, \tilde{\Theta}_{j}\right\rangle+\frac{1}{3}\left\langle R(\Phi, \tilde{\Theta}) \Phi, \tilde{\Theta}_{j}\right\rangle+\mathcal{O}\left(\varepsilon^{2}\right) \\
& +\varepsilon^{2} L(w, \Phi)+\varepsilon Q(w, \Phi),
\end{aligned}
$$

These follow from (3.3) together with the fact that $\left\langle\Upsilon, Z_{a}\right\rangle=0$ and $\left\langle\Upsilon, Z_{j}\right\rangle=0$ on $K_{\varepsilon}$.

Using Proposition 3.1, and some algebraic calculations, one can obtain

$$
\beta^{c}=w_{c}+\left\langle\Phi_{c}, \tilde{\Theta}\right\rangle+\varepsilon \Theta^{n+1} h(\tilde{\Theta})^{c}+\mathcal{O}\left(\varepsilon^{2}\right)+\varepsilon L(w, \Phi)+Q(w, \Phi) .
$$

and

$$
\begin{aligned}
\alpha^{j}\left\langle\Theta_{j}, \Theta_{i}\right\rangle= & w_{i}+\varepsilon \Theta^{n+1}\left\langle h(\tilde{\Theta}), \tilde{\Theta}_{i}\right\rangle+\varepsilon \Theta^{n+1}\langle h(\tilde{\Theta}), \tilde{\Theta}\rangle w_{i} \\
& -2 \varepsilon \Theta^{n+1}\left(\left\langle h\left(\tilde{\Theta}_{l}\right), \tilde{\Theta}_{i}\right\rangle w_{l}+h\left(\tilde{\Theta}_{i}\right)^{a} w_{a}+h\left(\tilde{\Theta}_{i}\right)^{a}\left\langle\Phi_{a}, \tilde{\Theta}\right\rangle\right) \\
& +\frac{1}{3} \varepsilon\left\langle R(\Phi, \tilde{\Theta}) \tilde{\Theta}, \tilde{\Theta}_{i}\right\rangle-\varepsilon \Theta^{n+1} h(\tilde{\Theta})^{a}\left\langle\Phi_{a}, \tilde{\Theta}_{i}\right\rangle \\
& -2 w w_{i}-w_{a}\left\langle\Phi_{a}, \tilde{\Theta}_{i}\right\rangle-\left\langle\Phi_{a}, \tilde{\Theta}\right\rangle\left\langle\Phi_{a}, \tilde{\Theta}_{i}\right\rangle \\
& +\frac{1}{3}\left\langle R(\Phi, \tilde{\Theta}) \Phi, \tilde{\Theta}_{i}\right\rangle+\mathcal{O}\left(\varepsilon^{2}\right)+\varepsilon^{2} L(w, \Phi)+\varepsilon Q(w, \Phi)
\end{aligned}
$$

Using these and the fact that $\left\langle\Theta_{j}, \Theta_{i}\right\rangle=\mu^{2} \delta_{i j}$, straightforward computations imply

$$
\begin{aligned}
|\tilde{N}|^{-1}= & 1+\varepsilon \Theta^{n+1}\left(\frac{1}{\mu^{2}}\left\langle h(\tilde{\Theta}), \tilde{\Theta}_{i}\right\rangle w_{i}+h(\tilde{\Theta})^{c} w_{c}+h(\tilde{\Theta})^{c}\left\langle\Phi_{c}, \tilde{\Theta}\right\rangle\right) \\
& +\frac{1}{6}\langle R(\Phi, \tilde{\Theta}) \Phi, \tilde{\Theta}\rangle+\frac{1}{2}\left(w_{c}^{2}+\frac{1}{\mu^{2}} w_{j}^{2}+2 w_{c}\left\langle\Phi_{c}, \tilde{\Theta}\right\rangle+\left\langle\Phi_{c}, \tilde{\Theta}\right\rangle^{2}\right) \\
& +\mathcal{O}\left(\varepsilon^{2}\right)+\varepsilon^{2} L(w, \Phi)+\varepsilon Q(w, \Phi) .
\end{aligned}
$$

The unit normal to the perturbed geodesic tube is then given simply by $N=\frac{\tilde{N}}{|\tilde{N}|}$. We summarize this in the following lemma

Proposition 3.2. The normal vector field $N$ to $S_{\varepsilon}(w, \Phi)$ is given by $N=\frac{\tilde{N}}{|\tilde{N}|}$ where

$$
\tilde{N}:=-\Upsilon+\alpha^{j} Z_{j}+\beta^{c} Z_{c}
$$

and where the coefficients $\alpha^{j}$ and $\beta^{c}$ are given by formulas (3.15) and (3.14). 
Using the fact that $\left.\Theta^{n+1}\right|_{\partial B_{1}^{n}}=0$ we can easily deduce:

Lemma 3.3. The perpendicularity condition is given by

$$
\left\langle N, \mathcal{V}^{\varepsilon}\right\rangle=(-1+w) w_{j} z^{j}+\overline{\mathcal{O}}\left(\varepsilon^{2}\right)+\varepsilon^{2} \bar{L}(w, \Phi)+\varepsilon \bar{Q}(w, \Phi) \quad \text { on } \partial(S N K)_{+},
$$

Proof. Since $\left.\Theta^{n+1}\right|_{\partial B_{1}^{n}}=0$ it follows that $\left\langle\mathcal{V}^{\varepsilon},-\Upsilon+\beta^{c} Z_{c}\right\rangle=0$ on $\partial B_{1}^{n}$ on the other hand using the fact that $R\left(E_{i}, E_{i}\right)=0$ with $\left.\frac{\partial \tilde{\Theta}}{\partial \tau}\right|_{\partial B_{1}^{n}}=0$ (see Subsection 2.4$)$ we get

$\left\langle\alpha^{j} Z_{j}, \mathcal{V}^{\varepsilon}\right\rangle=(-1+w) w_{j} \Theta_{j}^{n+1}+\overline{\mathcal{O}}\left(\varepsilon^{2}\right)+\varepsilon^{2} \bar{L}(w, \Phi)+\varepsilon \bar{Q}(w, \Phi)$ on $\partial(S N K)_{+}$

The lemma now follows since $\Theta_{j}^{n+1}=-\mu \Theta^{j}=-\mu^{2} z^{j}$ and $\left.\mu\right|_{\partial B_{1}^{n}}=1$.

\subsubsection{The second fundamental form}

In this paragraph we expand the coefficients of the second fundamental form. Recall that $\nabla$ is the Levi-Civita connection on $\partial \Omega$ and $h$ its second fundamental form, the derivation for vector fields on $\partial \Omega$ yields

$$
\begin{aligned}
\frac{\partial}{\partial z^{i}} X_{\alpha}\left(y,(1+w(y, z)) \tilde{\Theta}+\varepsilon^{-1} \Phi(\varepsilon y)\right)= & \varepsilon\left(w_{i} \Theta^{l}+(1+w) \Theta_{i}^{l}\right)\left(\nabla_{X_{l}} X_{\alpha}-h_{l \alpha} \mathcal{V}^{\varepsilon}\right), \\
\frac{\partial}{\partial y^{a}} X_{\alpha}\left(y,(1+w(y, z)) \tilde{\Theta}+\varepsilon^{-1} \Phi(\varepsilon y)\right)= & \varepsilon \delta_{a b}\left(\nabla_{X_{b}} X_{\alpha}-h_{b \alpha} \mathcal{V}^{\varepsilon}\right) \\
& +\varepsilon\left(w_{a} \Theta^{l}+\Phi_{a}^{l}\right)\left(\nabla_{X_{l}} X_{\alpha}-h_{l \alpha} \mathcal{V}^{\varepsilon}\right)
\end{aligned}
$$

Proposition 3.4. The following expansions hold

$$
\begin{aligned}
\left\langle N, \frac{\partial}{\partial y^{a}} Z_{a}\right\rangle= & -\varepsilon \Gamma_{a}^{a}(\tilde{\Theta})+\varepsilon \Theta^{n+1} h_{a a}-w_{a a}-\varepsilon\left\langle\Phi_{a a}, \tilde{\Theta}\right\rangle \\
& -\varepsilon\left\langle R\left(\Phi, E_{a}\right) E_{a}, \tilde{\Theta}\right\rangle+\varepsilon \Gamma_{a}^{c}(\tilde{\Theta}) \Gamma_{c}^{a}(\Phi) \\
& -2 \varepsilon \Theta^{n+1} w_{a} h(\tilde{\Theta})^{a}+\frac{\varepsilon}{\mu^{2}} w_{l}\left(\Gamma_{a}^{a}\left(\tilde{\Theta}_{l}\right)-h_{a a} \Theta_{l}^{m+1}\right) \\
& +\mathcal{O}\left(\varepsilon^{2}\right)+\varepsilon^{2} L(w, \Phi)+\varepsilon Q(w, \Phi)
\end{aligned}
$$




$$
\begin{aligned}
& \left\langle N, \frac{\partial}{\partial z^{j}} Z_{j}\right\rangle=\mu^{2}(1+w)-w_{j j}-\varepsilon \Theta^{n+1}\langle h(\tilde{\Theta}), \tilde{\Theta}\rangle w_{j j}-2 \varepsilon \Theta_{j}^{n+1}\langle h(\tilde{\Theta}), \tilde{\Theta}\rangle w_{j} \\
& +\varepsilon(1+2 w)\left(\Theta^{n+1}\left\langle h\left(\tilde{\Theta}_{j}\right), \tilde{\Theta}_{j}\right\rangle-2 \Theta_{j}^{n+1}\left\langle h(\tilde{\Theta}), \tilde{\Theta}_{j}\right\rangle-\Theta^{n+1}\left\langle h(\tilde{\Theta}), \tilde{\Theta}_{j j}\right\rangle\right) \\
& +\frac{\varepsilon}{\mu^{2}} w_{k}\left(2 \Theta^{n+1}\left\langle h\left(\tilde{\Theta}_{k}\right), \tilde{\Theta}_{i i}\right\rangle+2 \Theta_{i}^{n+1}\left\langle h\left(\tilde{\Theta}_{k}\right), \tilde{\Theta}_{i}\right\rangle+\Theta_{k}^{n+1}\left\langle h\left(\tilde{\Theta}_{i}\right), \tilde{\Theta}_{i}\right\rangle\right) \\
& +\frac{2}{3} \varepsilon\left\langle R\left(\Phi, \tilde{\Theta}_{j}\right) \tilde{\Theta}, \tilde{\Theta}_{j}\right\rangle-\frac{\varepsilon}{3}\left\langle R(\Phi, \tilde{\Theta}) \tilde{\Theta}, \tilde{\Theta}_{j j}\right\rangle \\
& +2 \varepsilon w_{c}\left(\Theta_{j}^{n+1} h\left(\tilde{\Theta}_{j}\right)^{c}+\Theta^{n+1} h\left(\tilde{\Theta}_{j j}\right)^{c}\right) \\
& +2 \varepsilon\left\langle\Phi_{\bar{c}}, \tilde{\Theta}\right\rangle\left(\Theta_{j}^{n+1} h\left(\tilde{\Theta}_{j}\right)^{c}+\Theta^{n+1} h\left(\tilde{\Theta}_{j j}\right)^{c}\right) \\
& +\varepsilon \Theta^{n+1} h(\tilde{\Theta})^{c}\left(\left\langle\Phi_{c}, \tilde{\Theta}_{j j}\right\rangle+\mu^{2}\left\langle\Phi_{c}, \tilde{\Theta}\right\rangle\right) \\
& +\varepsilon \Theta^{n+1} h(\tilde{\Theta})^{c}\left(w_{c}\left\langle\tilde{\Theta}, \tilde{\Theta}_{j j}\right\rangle+\mu^{2} w_{c}\right)-\frac{1}{6} \mu^{2}\langle R(\Phi, \tilde{\Theta}) \Phi, \tilde{\Theta}\rangle \\
& -\frac{1}{3}\left\langle R(\Phi, \tilde{\Theta}) \Phi, \tilde{\Theta}_{j j}\right\rangle-\frac{1}{2} \mu^{2} w_{c}^{2}+\frac{1}{2} \mu^{2}\left|\left\langle\Phi_{c}, \tilde{\Theta}\right\rangle\right|^{2} \\
& -\frac{1}{2} w_{k}^{2}+2 w_{j}^{2}+\left\langle\Phi_{c}, \tilde{\Theta}_{j j}\right\rangle w_{c}+\left\langle\Phi_{c}, \tilde{\Theta}\right\rangle\left\langle\Phi_{c}, \tilde{\Theta}_{j j}\right\rangle \\
& +(1+2 w) \alpha^{k}\left\langle\Theta_{j j}, \Theta_{k}\right\rangle+\mathcal{O}\left(\varepsilon^{2}\right)+\varepsilon^{2} L(w, \Phi)+\varepsilon Q(w, \Phi) ; \\
& \left.\left\langle N, \frac{\partial}{\partial y^{a}} Z_{b}\right\rangle=-\Gamma_{a}^{b}(\tilde{\Theta})+\varepsilon \Theta^{n+1} h_{a b}-w_{a b}+\mathcal{O}\left(\varepsilon^{2}\right)+\varepsilon L(w, \Phi)+Q(w, \Phi)\right) \quad a \neq b \\
& \left\langle N, \frac{\partial}{\partial y_{a}} Z_{j}\right\rangle=\varepsilon \Theta_{j}^{n+1} h(\tilde{\Theta})^{a}+\varepsilon \Theta^{n+1} h\left(\tilde{\Theta}_{j}\right)^{a}-w_{a j}+\mathcal{O}\left(\varepsilon^{2}\right)+\varepsilon L(w, \Phi)+Q(w, \Phi) \\
& \left\langle N, \frac{\partial}{\partial z_{i}} Z_{j}\right\rangle=-w_{i j}-\varepsilon \Theta_{i}^{n+1}\left\langle h(\tilde{\Theta}), \tilde{\Theta}_{j}\right\rangle-\varepsilon \Theta_{j}^{n+1}\left\langle h(\tilde{\Theta}), \tilde{\Theta}_{i}\right\rangle \\
& +\varepsilon \Theta^{n+1}\left\langle h\left(\tilde{\Theta}_{i}\right), \tilde{\Theta}_{j}\right\rangle-\varepsilon \Theta^{n+1}\left\langle h(\tilde{\Theta}), \tilde{\Theta}_{i j}\right\rangle \\
& +\alpha^{k}\left\langle\Theta_{i j}, \Theta_{k}\right\rangle+\mathcal{O}\left(\varepsilon^{2}\right)+\varepsilon L(w, \Phi)+Q(w, \Phi), \quad i \neq j
\end{aligned}
$$


Proof. The proof is similar in spirit to the one of [10, Proposition 3.3]. So we will be sketchy here referring to the aforementioned paper for more details. We have that

$$
\begin{aligned}
\frac{\partial}{\partial y^{a}} Z_{a}= & \varepsilon\left(\nabla_{X_{a}} X_{a}-h_{a a} \mathcal{V}^{\varepsilon}\right)+w_{a a} \Upsilon+2 \Theta^{n+1} w_{a} D_{a} \mathcal{V}^{\varepsilon}+\varepsilon \Phi_{a a}^{l} X_{l} \\
& +\Theta^{n+1} D_{a} D_{a} \mathcal{V}^{\varepsilon}+\left(\mathcal{O}\left(\varepsilon^{2}\right)+\varepsilon^{2} L(w, \Phi)+\varepsilon Q(w, \Phi)\right) X_{\alpha} \\
& +\left(\mathcal{O}\left(\varepsilon^{2}\right)+\varepsilon^{2} L(w, \Phi)+\varepsilon Q(w, \Phi)\right) \mathcal{V}^{\varepsilon}
\end{aligned}
$$

and for $a \neq b$

$$
\begin{aligned}
\frac{\partial}{\partial y^{a}} Z_{b}= & \varepsilon\left(\nabla_{X_{b}} X_{a}-h_{a b} \mathcal{V}^{\varepsilon}\right)+w_{a b} \Upsilon \\
& +\left(\mathcal{O}\left(\varepsilon^{2}\right)+\varepsilon L(w, \Phi)+Q(w, \Phi)\right) X_{\alpha} \\
& +\left(\mathcal{O}\left(\varepsilon^{2}\right)+\varepsilon^{2} L(w, \Phi)+\varepsilon Q(w, \Phi)\right) \mathcal{V}^{\varepsilon} \\
\frac{\partial}{\partial z^{i}} Z_{i}= & w_{i i} \Upsilon+2 w_{i} \Upsilon_{i}+2 \varepsilon \Theta^{l} \Theta_{i}^{s} w_{i}\left(\nabla_{X_{s}} X_{l}-h_{s l} \mathcal{V}^{\varepsilon}\right)+2 \Theta^{n+1} D_{i} \mathcal{V}^{\varepsilon} w_{i} \\
& +(1+w) \Upsilon_{i i}+(1+w)\left(2 \Theta_{i}^{n+1} D_{i} \mathcal{V}^{\varepsilon}+\Theta^{n+1} D_{i} D_{i} \mathcal{V}\right) \\
& +\varepsilon(1+2 w) \Theta_{i}^{l} \Theta_{i}^{s}\left(\nabla_{X_{s}} X_{l}-h_{s l} \mathcal{V}^{\varepsilon}\right) \\
& +\left(\mathcal{O}\left(\varepsilon^{2}\right)+\varepsilon^{2} L(w, \Phi)+\varepsilon Q(w, \Phi)\right) X_{\alpha} \\
& +\left(\mathcal{O}\left(\varepsilon^{2}\right)+\varepsilon^{2} L(w, \Phi)+\varepsilon Q(w, \Phi)\right) \mathcal{V}^{\varepsilon}
\end{aligned}
$$

and for $i \neq j$

$$
\begin{aligned}
\frac{\partial}{\partial z^{i}} Z_{j}= & w_{i j} \Upsilon+w_{i} \Upsilon_{j}+w_{j} \Upsilon_{i}+\Theta_{i}^{n+1} D_{j} \mathcal{V}^{\varepsilon}+\Theta_{j}^{n+1} D_{i} \mathcal{V}^{\varepsilon}+(1+w) \Upsilon_{i j} \\
& +\varepsilon \Theta_{i}^{l} \Theta_{j}^{s}\left(\nabla_{X_{s}} X_{l}-h_{s l} \mathcal{V}^{\varepsilon}\right) \\
& +\Theta^{n+1} D_{i} D_{j} \mathcal{V}^{\varepsilon}+\left(\mathcal{O}\left(\varepsilon^{2}\right)+\varepsilon L(w, \Phi)+Q(w, \Phi)\right) X_{\alpha} \\
& +\left(\mathcal{O}\left(\varepsilon^{2}\right)+\varepsilon^{2} L(w, \Phi)+\varepsilon Q(w, \Phi)\right) \mathcal{V}^{\varepsilon} .
\end{aligned}
$$


Finally

$$
\begin{aligned}
\frac{\partial}{\partial y^{a}} Z_{j}=\frac{\partial}{\partial z_{j}} Z_{a}= & \varepsilon \Theta_{j}^{s}\left(\nabla_{X_{s}} X_{a}-h_{a s} \mathcal{V}^{\varepsilon}\right)+w_{a j} \Upsilon+w_{a} \Upsilon_{j}+\Theta_{j}^{n+1} D_{a} \mathcal{V}^{\varepsilon} \\
& +\left(\mathcal{O}\left(\varepsilon^{2}\right)+\varepsilon^{2} L(w, \Phi)+\varepsilon Q(w, \Phi)\right) X_{\alpha} \\
& +\left(\mathcal{O}\left(\varepsilon^{2}\right)+\varepsilon^{2} L(w, \Phi)+\varepsilon Q(w, \Phi)\right) \mathcal{V}^{\varepsilon}
\end{aligned}
$$

Recalling the expansions, see [10, Lemma 2.1].

$$
\begin{aligned}
& \nabla_{X_{i}} X_{j}=(\mathcal{O}(\varepsilon)+L(w, \Phi)+Q(w, \Phi)) X_{\gamma}, \\
& \nabla_{X_{a}} X_{i}=-\Gamma_{a}^{b}\left(E_{i}\right) X_{b}+(\mathcal{O}(\varepsilon)+L(w, \Phi)+Q(w, \Phi)) X_{\gamma}
\end{aligned}
$$

We will also need the following expansion which follows from the result of [10, Lemma 2.2] (with obvious modifications).

$$
\begin{aligned}
& \nabla_{X_{a}} X_{b}=\Gamma_{a}^{b}\left(E_{j}\right) X_{j}-\left\langle R\left(\varepsilon \tilde{\Theta}+\Phi, E_{a}\right) E_{j}, E_{b}\right\rangle X_{j} \\
& +\frac{1}{2}\left(\left\langle R\left(E_{a}, E_{b}\right)(\varepsilon \tilde{\Theta}+\Phi), E_{j}\right\rangle-\Gamma_{a}^{c}(\varepsilon \tilde{\Theta}+\Phi) \Gamma_{c}^{b}\left(E_{j}\right)-\Gamma_{c}^{b}(\varepsilon \tilde{\Theta}+\Phi) \Gamma_{a}^{c}\left(E_{j}\right)\right) X_{j} \\
& +(\mathcal{O}(\varepsilon)+L(w, \Phi)+Q(w, \Phi)) X_{c}+\left(\mathcal{O}\left(\varepsilon^{2}\right)+\varepsilon L(w, \Phi)+Q(w, \Phi)\right) X_{j} .
\end{aligned}
$$

These implies in particular

$$
\begin{aligned}
\left\langle\Upsilon, \nabla_{X_{a}} X_{a}\right\rangle= & \Theta^{l} \Gamma_{a}^{a}\left(E_{i}\right)\left(\delta_{l i}+2 \varepsilon \Theta^{n+1} h_{l i}\right)-\varepsilon\left\langle R\left(\tilde{\Theta}, E_{a}\right) \tilde{\Theta}, E_{a}\right\rangle \\
& -\left\langle R\left(\tilde{\Theta}, E_{a}\right) \Phi, E_{a}\right\rangle-\varepsilon \Gamma_{a}^{c}(\tilde{\Theta}) \Gamma_{c}^{a}(\tilde{\Theta}) \\
& -\Gamma_{a}^{c}(\tilde{\Theta}) \Gamma_{c}^{a}(\Phi)+\mathcal{O}\left(\varepsilon^{2}\right)+\varepsilon L(w, \Phi)+Q(w, \Phi) .
\end{aligned}
$$

On the other hand we have that

$$
\begin{aligned}
D_{a} D_{a} \mathcal{V}^{\varepsilon}= & \varepsilon w_{a a} h(\tilde{\Theta})^{\alpha} X_{\alpha}+\left(\mathcal{O}\left(\varepsilon^{2}\right)+\varepsilon^{2} L(w, \Phi)+\varepsilon Q(w, \Phi)\right) X_{\beta} \\
& +\left(\mathcal{O}\left(\varepsilon^{2}\right)+\varepsilon^{2} L(w, \Phi)+\varepsilon Q(w, \Phi)\right) \mathcal{V}^{\varepsilon}
\end{aligned}
$$

which implies

$$
\left\langle D_{a} D_{a} \mathcal{V}^{\varepsilon}, \Upsilon\right\rangle=\varepsilon w_{a a}\langle h(\tilde{\Theta}), \tilde{\Theta}\rangle+\mathcal{O}\left(\varepsilon^{2}\right)+\varepsilon^{2} L(w, \Phi)+\varepsilon Q(w, \Phi) .
$$

Using these together with (3.14), (3.15) and Lemma 2.2, the first estimate follows at once. For the other estimates one can proceed similarly. 


\subsubsection{The mean curvature of perturbed tubes}

Collecting the estimates of the last subsection we obtain the expansion of the mean curvature of the hypersurface $S_{\varepsilon}(w, \Phi)$. In the coordinate system defined in the previous sections, we get

$$
\begin{aligned}
m H(w, \Phi)= & n-\varepsilon \Gamma_{a}^{a}(\tilde{\Theta})+\varepsilon \Theta^{n+1} h_{a a}+\varepsilon \Theta^{n+1}\left[(n+3)\langle h(\tilde{\Theta}), \tilde{\Theta}\rangle-h_{j j}\right] \\
& +\mathcal{O}\left(\varepsilon^{2}\right)-\left(\Delta_{K_{\varepsilon}} w+\Delta_{S^{n}} w+n w\right) \\
& -\varepsilon\left(\left\langle\Delta_{K} \Phi+R\left(\Phi, E_{a}\right) E_{a}, \tilde{\Theta}\right\rangle-\Gamma_{a}^{c}(\Phi) \Gamma_{c}^{a}(\tilde{\Theta})\right) \\
& -\varepsilon \Theta^{n+1}\langle h(\tilde{\Theta}), \tilde{\Theta}\rangle \Delta_{S^{n}} w-2 \varepsilon(n+3) \Theta^{n+1}\left\langle h(\tilde{\Theta}), \nabla_{S^{n}} w\right\rangle \\
& +2 \varepsilon \Theta^{n+1} \nabla_{S^{n}}^{2} w: h-\varepsilon\left(\langle h(\tilde{\Theta}), \tilde{\Theta}\rangle+h_{j j}+h_{a a}\right)\left\langle\nabla_{S^{n}} w, E_{n+1}\right\rangle \\
& -(1+3 n) \varepsilon \Theta^{n+1} h(\tilde{\Theta})^{a} w_{a}-2 \varepsilon \Theta^{n+1} h\left(\nabla_{S^{n}} w_{a}\right)^{a}+\varepsilon \Gamma_{a}^{a}\left(\nabla_{S^{n}} w\right) \\
& -2 \varepsilon \nabla_{K_{\varepsilon}}^{2} w: \Gamma(\tilde{\Theta})+2 \varepsilon \Theta^{n+1} h_{a a} w_{a a}-(3 n+1) \varepsilon \Theta^{n+1} h(\tilde{\Theta})^{a}\left\langle\Phi_{\bar{a}}, \tilde{\Theta}\right\rangle \\
& +\varepsilon \Theta^{n+1} h\left(\Phi_{\bar{a}}\right)^{a}+2 \varepsilon \Theta^{n+1} h: \Gamma(\Phi) \\
& +n w^{2}+\frac{2-n}{2}\left|\nabla_{S^{n}} w\right|^{2}+2 w \Delta_{S^{n}} w-\frac{n}{2}\left(w_{a}+\left\langle\Phi_{\bar{a}}, \tilde{\Theta}\right\rangle\right)^{2} \\
& -\left\langle\Phi_{\bar{a}}, \nabla_{S^{n}} w_{a}\right\rangle-2 \nabla_{K_{\varepsilon}}^{2} w: \Gamma(\Phi)+\frac{n+2}{6}\langle R(\Phi, \tilde{\Theta}) \Phi, \tilde{\Theta}\rangle \\
& -\frac{1}{3}\left\langle R\left(\Phi, E_{i}\right) \Phi, E_{i}\right\rangle+\mathcal{O}\left(\varepsilon^{2}\right)+\varepsilon^{2} L(w, \Phi)+\varepsilon Q(w, \Phi) .
\end{aligned}
$$

Here we have used the formulas in Lemma 2.4, the fact that

$$
\Delta_{S^{n}}=\frac{1}{\mu^{2}}\left(\Delta_{\mathbb{R}^{n}}-\left\langle\Theta_{i i}, \Theta_{k}\right\rangle \partial_{k}\right) .
$$

and the notation $A: B=A_{s t} B_{s t}$ for two linear operators $A$ and $B$. Where summation over repeated indices is understood.

Let us emphasize the use of the variables $y_{\bar{a}}=\varepsilon y_{a}$ on $K$. With an abuse of notation, we call $w$ the function $\bar{w}(\bar{y})=w(y)=w\left(\varepsilon^{-1} \bar{y}\right)$ defined on $K$ so that $\varepsilon w_{\bar{a}}=w_{a}$ and $\varepsilon^{2} w_{\bar{a} \bar{a}}=w_{a a}$. We first define the following operators appearing in 
the above expansion

$$
\begin{aligned}
\mathcal{L}^{1}(w):= & -\langle h(\tilde{\Theta}), \tilde{\Theta}\rangle \Delta_{S^{n}} w-2(n+3) \Theta^{n+1}\left\langle h(\tilde{\Theta}), \nabla_{S^{n}} w\right\rangle \\
& +2 \Theta^{n+1} \nabla_{S^{n}}^{2} w: h-\left(\langle h(\tilde{\Theta}), \tilde{\Theta}\rangle+h_{j j}+h_{a a}\right)\left\langle\nabla_{S^{n}} w, E_{n+1}\right\rangle \\
& -\varepsilon(1+3 n) \Theta^{n+1}\left\langle h(\tilde{\Theta}), \nabla_{K} w\right\rangle+\varepsilon \Theta^{n+1} h\left(\nabla_{S^{n}} w_{\bar{a}}\right)^{a} \\
& -2 \varepsilon^{2} \nabla_{K}^{2} w: \Gamma(\tilde{\Theta})+2 \varepsilon^{2} \Theta^{n+1} h_{a a} w_{\bar{a} \bar{a}}, \\
\mathcal{J}^{1} \Phi:=- & (3 n+1) \Theta^{n+1} h(\tilde{\Theta})^{a}\left\langle\Phi_{\bar{a}}, \tilde{\Theta}\right\rangle+\Theta^{n+1} h\left(\Phi_{\bar{a}}\right)^{a}+2 \Theta^{n+1} h: \Gamma(\Phi),
\end{aligned}
$$

and the quadratic term

$$
\begin{aligned}
\mathcal{Q}^{1}(w, \Phi):= & n w^{2}+\frac{2-n}{2}\left|\nabla_{S^{n}} w\right|^{2}+2 w \Delta_{S^{n}} w-\frac{n}{2}\left(\varepsilon w_{\bar{a}}+\left\langle\Phi_{\bar{a}}, \tilde{\Theta}\right\rangle\right)^{2} \\
& -\varepsilon\left\langle\Phi_{\bar{a}}, \nabla_{S^{n}} w_{\bar{a}}\right\rangle-2 \varepsilon^{2} \nabla_{K}^{2} w: \Gamma(\Phi) \\
& +\frac{n+2}{6}\langle R(\Phi, \tilde{\Theta}) \Phi, \tilde{\Theta}\rangle-\frac{1}{3}\left\langle R\left(\Phi, E_{i}\right) \Phi, E_{i}\right\rangle
\end{aligned}
$$

Next, we define

$$
\mathcal{L}_{\varepsilon}:=\varepsilon^{2} \Delta_{K}+\Delta_{S^{n}}+n, \quad \mathcal{L}_{0}:=\Delta_{S^{n}}+n
$$

and the Jacobi operator about $K$ in $(\partial \Omega, \bar{g})$, see Subsection 2.2

$$
\mathfrak{J}:=\Delta^{\perp}-\mathcal{R}^{\perp}+\mathcal{B} .
$$

Recall that (see Subsection 2.4) the outer unit normal to the boundary of $\partial S_{+}^{n}$ in $S_{+}^{n}$ is $\eta=-E_{n+1}$,

$$
\frac{\partial w}{\partial \eta}=-\left\langle\nabla_{S_{+}^{n}} w, E_{n+1}\right\rangle .
$$

Using these definitions, we obtain the following result:

Proposition 3.5. Assume that $K$ is a minimal submanifold, then the mean curvature of $S_{\varepsilon}(w, \Phi)$ can be expanded as

$$
\begin{aligned}
m H(w, \Phi)= & n+\varepsilon \Theta^{n+1} h_{a a}+\varepsilon \Theta^{n+1}\left[(n+3)\langle h(\tilde{\Theta}), \tilde{\Theta}\rangle-h_{j j}\right]+\mathcal{O}\left(\varepsilon^{2}\right) \\
& -\mathcal{L}_{\varepsilon} w-\varepsilon\langle\mathfrak{J} \Phi, \tilde{\Theta}\rangle+\varepsilon \mathcal{L}^{1} w+\varepsilon \mathcal{J}^{1}(\Phi)+\mathcal{Q}^{1}(w, \Phi) \\
& +\varepsilon^{2} L(w, \Phi)+\varepsilon Q(w, \Phi) \quad \text { in } S_{\varepsilon}(w, \Phi) .
\end{aligned}
$$


where $\mathcal{L}^{1}$ is defined in (3.22), $\mathcal{J}^{1}$ is given in (3.23), while $\mathcal{Q}^{1}$ is a quadratic term defined in (3.24). Moreover, the orthogonality condition is equivalent to the following boundary condition on the function $w$ :

$$
\left\langle N, \mathcal{V}^{\varepsilon}\right\rangle=-\frac{\partial w}{\partial \eta}+w \frac{\partial w}{\partial \eta}+\overline{\mathcal{O}}\left(\varepsilon^{2}\right)+\varepsilon^{2} \bar{L}(w, \Phi)+\varepsilon \bar{Q}(w, \Phi) \quad \text { on } \partial S_{\varepsilon}(w, \Phi) .
$$

Proof. The expression of the mean curvature can be obtained rather easily taking into account the above definitions (with obvious modifications) and the minimality of $K$ which implies

$$
\Gamma_{a}^{a}=0 .
$$

With these notations finding $w$ and $\Phi$ such that the equation $m H=n$ and $\left\langle N, \mathcal{V}^{\varepsilon}\right\rangle=0$ hold is equivalent to solve

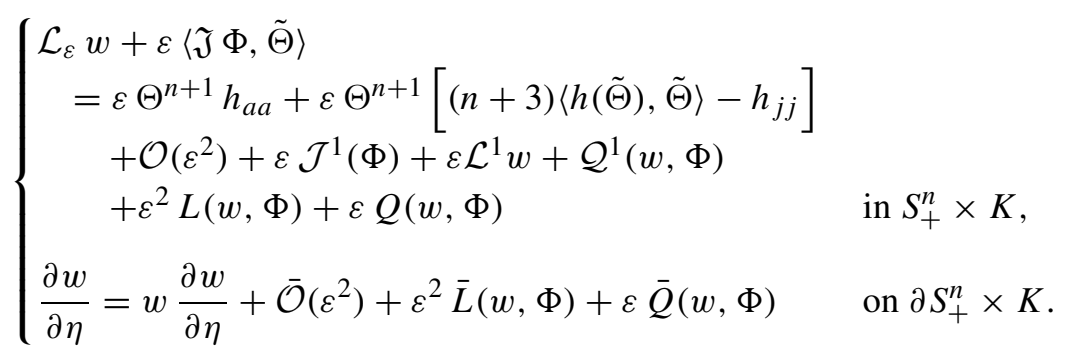

\section{Adjusting the tube $\bar{S}_{\varepsilon}\left(K_{\varepsilon}\right)$}

In this section we annihilate the error terms $(\mathcal{O}(\varepsilon)$ ) appearing in (3.26) at any given order. The non-degeneracy of the submanifold $K$ will play a crucial role in such a construction. We denote by $\Pi$ the $L^{2}$ projection on the subspace spanned by the $\Theta^{i}, i=1, \cdots, n$ and set $(S N K)_{+}:=S_{+}^{n} \times K$.

We set

$$
\hat{w}^{(r)}=\sum_{d=1}^{r} \varepsilon^{d} w^{(d)} \quad \text { and } \quad \hat{\Phi}^{r}=\sum_{d=1}^{r-1} \varepsilon^{d} \Phi^{(d)} .
$$

\section{Construction of $w^{(1)}$}

We first want to kill the term $\mathcal{O}(\varepsilon)$. This is equivalent to have

$$
\begin{cases}m H\left(\hat{w}^{(r)}, \hat{\Phi}^{(r)}\right)=n+\mathcal{O}\left(\varepsilon^{2}\right) & \text { in } S_{\varepsilon}\left(\hat{w}^{(r)}, \hat{\Phi}^{(r)}\right), \\ \left\langle N, \mathcal{V}^{\varepsilon}\right\rangle=\overline{\mathcal{O}}\left(\varepsilon^{2}\right) & \text { on } \partial S_{\varepsilon}\left(\hat{w}^{(r)}, \hat{\Phi}^{(r)}\right) .\end{cases}
$$


This gives the following equation in $w^{(1)}$

$$
\begin{aligned}
\mathcal{L}_{0} w^{(1)} & =\Theta^{n+1} h_{a a}+\Theta^{n+1}\left[(n+3)\langle h(\tilde{\Theta}), \tilde{\Theta}\rangle-h_{j j}\right] \quad \text { in }(S N K)_{+} ; \\
\frac{\partial w^{(1)}}{\partial \eta} & =0 \quad \text { on } \partial(S N K)_{+} .
\end{aligned}
$$

By the result from Subsection 2.4 (with $\gamma=\frac{\pi}{2}$ ) and Fredholm alternative theorem, the solvability of the above system is possible provided

$\int_{S_{+}^{n}}\left(\Theta^{n+1} h_{a a}+\Theta^{n+1}\left[(n+3)\langle h(\tilde{\Theta}), \tilde{\Theta}\rangle-h_{j j}\right]\right) \Theta^{i} d \theta=0 \quad$ for all $i=1, \cdots, n$

which is the case by oddness, here $d \theta$ denotes the volume element on $S_{+}^{n}$.

Notice that the variable $\bar{y}$ is being considered as a parameter so that $w^{(1)}$ is as smooth as the right-hand side in this variable.

\section{Constructing $w^{(2)}$}

We turn now to the term of order $\varepsilon^{2}$. We have

$$
\begin{cases}m H\left(\hat{w}^{(r)}, \hat{\Phi}^{(r)}\right)=n+\mathcal{O}\left(\varepsilon^{3}\right) & \text { in } S_{\varepsilon}\left(\hat{w}^{(r)}, \hat{\Phi}^{(r)}\right) \\ \left\langle N, \mathcal{V}^{\varepsilon}\right\rangle=\overline{\mathcal{O}}\left(\varepsilon^{2}\right) & \text { on } \partial S_{\varepsilon}\left(\hat{w}^{(r)}, \hat{\Phi}^{(r)}\right)\end{cases}
$$

Since the terms involving $\Phi$ in $\mathcal{Q}^{1}\left(\varepsilon w^{(1)}, \varepsilon \Phi^{(1)}\right)$ are of the form $\varepsilon^{3} L\left(\Phi^{(1)}\right)$ and $Q\left(\hat{\Phi}^{(r)}, \hat{\Phi}^{(r)}\right)$, we are led to a system in $w^{(2)}$ and $\Phi^{(1)}$ given by

$$
\begin{aligned}
\mathcal{L}_{0} w^{(2)} & =\left\langle\mathfrak{J} \Phi^{(1)}, \tilde{\Theta}\right\rangle+\mathcal{O}(1)+\mathcal{L}^{1} w^{(1)}+\mathcal{J}^{1}\left(\Phi^{(1)}\right)+Q\left(\hat{\Phi}^{(r)}, \hat{\Phi}^{(r)}\right) \text { in }(S N K)_{+} \\
\frac{\partial w^{(2)}}{\partial \eta} & =\overline{\mathcal{O}}(1) \quad \text { on } \partial(S N K)_{+} \cdot
\end{aligned}
$$

Note that $\Pi \mathcal{J}^{1}=0$ and $\Pi Q\left(\Phi^{(1)}, \Phi^{(1)}\right)=0$ so the above problem is solvable if and only if

$$
\begin{aligned}
\int_{S_{+}^{n}}\left\langle\mathfrak{J} \Phi^{(1)}, \tilde{\Theta}\right\rangle \Theta^{i} d \theta & +\int_{S_{+}^{n}}\left(\mathcal{O}(1)+\mathcal{L}^{1} w^{(1)}\right) \Theta^{i} d \theta \\
& +\oint_{\partial S_{+}^{n}} \overline{\mathcal{O}}(1) \Theta^{i} d \bar{\theta}=0 \quad \text { for all } i=1 \cdots n
\end{aligned}
$$

where $d \theta$ and $d \bar{\theta}$ are the volume elements on $S_{+}^{n}$ and $\partial S_{+}^{n}$ respectively. This gives an equation on $\Phi^{(1)}$ which can be solved using the non degeneracy of the submanifold $K$ because in this case $\mathfrak{J}$ is invertible. Once this is done, we obtain readily $w^{(2)}$. 


\section{Constructing $w^{(r)}$}

We want to construct an approximate solution as accurate as possible, and to do so we will use an iterative scheme. Suppose the couple $\left(w^{(r-1)}, \Phi^{(r-2)}\right)$ is already determined. To find $\left(w^{(r)}, \Phi^{(r-1)}\right)$, it suffices to check that when we project on the Kernel of $\mathcal{L}_{0}$, the operator involving $\Phi^{(r-1)}$ should be only the invertible Jacobi operator $\mathfrak{J}$. This is the case since the only term that can bring $\Phi^{(r-1)}$ at this iteration step is $\mathcal{Q}_{r-1}^{1}(w, \Phi)$ which gives only terms of the form $\varepsilon^{2} \Phi$ and $Q\left(\hat{\Phi}^{(r)}, \hat{\Phi}^{(r)}\right)$ moreover $\Pi \mathcal{J}_{r-1}^{1}\left(\Phi^{(r-1)}\right)=\Pi Q\left(\hat{\Phi}^{(r)}, \hat{\Phi}^{(r)}\right)=0$.

The index $r$ appearing in the linear and quadratic terms means that they depend on the iteration step while the operator $\mathcal{J}_{r}^{1}$ keep its same properties because it is influenced only by the even quadratic terms in $Q\left(\hat{\Phi}^{(r)}+\Phi, \hat{\Phi}^{(r)}+\Phi\right)$ appearing in $Q^{1}\left(\hat{w}^{(r)}+w, \hat{\Phi}^{(r)}+\Phi\right)$.

By induction, in the same argument, for every $r \in \mathbb{N}$, we can find $\left(w^{(d)}, \Phi^{(d)}\right)$, $d=1, \cdots, r$ smooth such that

$$
\hat{w}^{(r)}=\sum_{d=1}^{r} \varepsilon^{d} w^{(d)}=\mathcal{O}(\varepsilon) \quad \text { and } \quad \hat{\Phi}^{(r)}=\sum_{d=1}^{r-1} \varepsilon^{d} \Phi^{(d)}=\mathcal{O}(\varepsilon)
$$

and that

$$
\begin{array}{ccc}
m H\left(\hat{w}^{(r)}, \hat{\Phi}^{(r)}\right)=n+\mathcal{O}\left(\varepsilon^{r+1}\right) & \text { in } \quad S_{\varepsilon}\left(\hat{w}^{(r)}, \hat{\Phi}^{(r)}\right), \\
\left\langle N, \mathcal{V}^{\varepsilon}\right\rangle=\overline{\mathcal{O}}\left(\varepsilon^{r+2}\right) \quad \text { on } \quad \partial S_{\varepsilon}\left(\hat{w}^{(r)}, \hat{\Phi}^{(r)}\right) .
\end{array}
$$

Remark 4.1. Notice that as in [11] we omitted the terms involving derivatives with respect to $\bar{y}$ of the function $w$ (by considering $\mathcal{L}_{0}$ instead of $\mathcal{L}_{\varepsilon}$ ), this is due to the fact that since $w$ is slow dependent on $y_{a}$, when differentiating with respect to $y_{\bar{a}}$ we pick up an $\varepsilon$ at each differentiation, this gives us smaller terms. However, when applying elliptic regularity theorems we might loose two derivatives at each iteration. This indeed is not a problem since one needs just a finite number of iterations. We refer the reader to [11], where a more explanation is given.

We are left to find $w$ and $\Phi$ such that

$$
\begin{array}{rll}
m H\left(\hat{w}^{(r)}+w, \hat{\Phi}^{r}+\Phi\right)=n & \text { in } & S_{\varepsilon}\left(\hat{w}^{(r)}+w, \hat{\Phi}^{r}+\Phi\right), \\
\left\langle N, \mathcal{V}^{\varepsilon}\right\rangle=0 & \text { on } & \partial S_{\varepsilon}\left(\hat{w}^{(r)}+w, \hat{\Phi}^{r}+\Phi\right) .
\end{array}
$$

We define the linearized mean curvature operator about $S_{\varepsilon}\left(\hat{w}^{r}, \hat{\Phi}^{r}\right)$

$$
\mathbb{L}_{\varepsilon, r}(w, \Phi)=\frac{1}{\varepsilon}\left(\mathcal{L}_{\varepsilon} w+\varepsilon \mathcal{L}_{r}^{1}(w)\right)+\langle\mathfrak{J} \Phi, \tilde{\Theta}\rangle+\mathcal{J}_{r}^{1}(\Phi)+\varepsilon L_{r}(w, \Phi) .
$$

The index $r$ appearing in the constant, linear and quadratic terms means that they depend on the iteration step but keep there properties. 
We notice that $\mathbb{L}_{\varepsilon, r}$ is not precisely the usual Jacobi operator because we are parameterizing this hypersurface as a graph over $S_{\varepsilon}\left(\hat{w}^{r}, \hat{\Phi}^{r}\right)$ using the vector field $-\Upsilon$ rather than the unit normal $N$.

Using Remark 2.3 (with $\gamma=\frac{\pi}{2}$ ), suppose that $\Sigma=S_{\varepsilon}\left(\hat{w}^{r}, \hat{\Phi}^{r}\right)$ and $\hat{N}=-\Upsilon$. From (4.1) and Proposition 3.2 we have

$$
\langle N,-\Upsilon\rangle=1+\mathcal{O}\left(\varepsilon^{2}\right) .
$$

Furthermore, from Proposition 3.1 and (4.1), the volume forms of the tubes $S_{\varepsilon}\left(\hat{w}^{r}, \hat{\Phi}^{r}\right)$ and $(S N K)_{+}$are related by

$$
d v o l_{S_{\varepsilon}\left(\hat{w}^{r}, \hat{\Phi}^{r}\right)}=(1+\mathcal{O}(\varepsilon)) d v o l_{(S N K)_{+}} .
$$

We define $\delta_{\varepsilon, r}>0$ by

$$
\langle N,-\Upsilon\rangle d v o l_{S_{\varepsilon}\left(\hat{w}^{r}, \hat{\Phi}^{r}\right)}=\delta_{\varepsilon, r} d v o l_{(S N K)_{+}} .
$$

Multiplying by $\delta_{\varepsilon, r}$, the system (4.2) will change the terms $\mathcal{L}_{r}^{1}, L_{r}, \bar{L}_{r}$, the constant and quadratic terms will keep there properties and there will be a new linear operator $\overline{\mathcal{L}}_{r}^{1}(w)$ on the boundary. We keep the same notations for these terms and call $\mathbb{L}_{\varepsilon, r}$ the new selfadjoint operator $\delta_{\varepsilon, r} \mathbb{L}_{\varepsilon, r}$ with respect to the standard $L^{2}(S N K)_{+}$-inner product.

Now since $\bar{L}_{r}(w, \Phi)$ and $\overline{\mathcal{L}}_{r}^{1}(w)$ involves only terms of the form $w, \partial_{z^{i}} w$, we may extend $\bar{L}_{r}(w, \Phi), \overline{\mathcal{L}}_{r}^{1}(w)$ and $\overline{\mathcal{O}}_{r}\left(\varepsilon^{r+1}\right)$ in $(S N K)_{+}$and this will just add some terms in $L_{r}(w, \Phi), \mathcal{L}_{r}^{1}(w)$ and $\mathcal{O}_{r}\left(\varepsilon^{r}\right)$ respectively which will maintain there properties.

Without loss of generality we may replace the solvability of (4.2) with the following equation.

$$
\begin{aligned}
\mathbb{L}_{\varepsilon, r}(w, \Phi) & =\frac{1}{\varepsilon} Q_{r}(w, \Phi)+\mathcal{O}_{r}\left(\varepsilon^{r}\right) & & \text { in }(S N K)_{+}, \\
\frac{\partial w}{\partial \eta} & =\frac{1}{\varepsilon} \bar{Q}_{r}(w, \Phi) & & \text { on } \partial(S N K)_{+} .
\end{aligned}
$$

We will try to invert the linear operator on the left-hand side and this will lead us to study the spectrum of the operator by selfadjointness.

\section{Spectral analysis}

\section{Function space}

Fix $\frac{1}{2}>s>0$. For any $v \in L^{2}(S N K)_{+}:=L^{2}\left(S_{+}^{n} \times K\right)$, set

$$
\langle\Phi, \tilde{\Theta}\rangle:=\Pi v, \quad \varepsilon^{-1+2 s} w:=\Pi^{\perp} v,
$$


so that

$$
v=\varepsilon^{1-2 s} w+\langle\Phi, \tilde{\Theta}\rangle .
$$

It will be understood that $\Phi^{i}$ for $i=1, \cdots, n$ are the components of $\Pi v$ on $N K$. Conversely if couple a $(w, \Phi) \in \Pi^{\perp} L^{2}(S N K)_{+} \times L^{2}(K, N K)$ is given, we associate to it a function $v$ as in (5.1).

Later we will often decompose

$$
w=w_{0}+w_{1}
$$

where $w_{0}$ is a function on $K$ and $w_{1}$ has zero mean value with respect to the angular integrals.

The volume element of $(S N K)_{+}=S_{+}^{n} \times K$ will be denoted by $d \theta d \bar{y}$.

As it will be apparent later, we will consider the following weighted Hilbert subspaces of $L^{2}(S N K)_{+}$

$$
\begin{aligned}
L_{\varepsilon}^{2}:=\left\{v=\varepsilon^{1-2 s} w+\langle\Phi, \tilde{\Theta}\rangle \in L^{2}(S N K)_{+} \quad: \quad\right. & \varepsilon^{-2 s} \int_{(S N K)_{+}}|w|^{2} d \theta d \bar{y} \\
& \left.+\int_{K}|\Phi|^{2} d \bar{y}<\infty\right\}
\end{aligned}
$$

with corresponding norm

$$
\|v\|_{L_{\varepsilon}^{2}}^{2}:=\varepsilon^{-2 s} \int_{(S N K)_{+}}|w|^{2} d \theta d \bar{y}+\int_{K}|\Phi|^{2} d \bar{y} .
$$

We also define

$$
\begin{aligned}
H_{\varepsilon}^{1}:=\left\{v \in L_{\varepsilon}^{2}: \quad\right. & \varepsilon^{-2 s} \int_{(S N K)_{+}}\left(\varepsilon^{2}\left|\nabla_{K} w\right|^{2}+\left|\nabla_{S_{+}^{n}} w\right|^{2}+|w|^{2}\right) d \theta d \bar{y} \\
& \left.+\int_{K}\left(\left|\nabla_{K} \Phi\right|^{2}+|\Phi|^{2}\right) d \bar{y}<\infty\right\}
\end{aligned}
$$

with corresponding norm

$$
\|v\|_{H_{\varepsilon}^{1}}^{2}:=\varepsilon^{-2 s} \int_{(S N K)_{+}}\left(\varepsilon^{2}\left|\nabla_{K} w\right|^{2}+\left|\nabla_{S_{+}^{n}} w\right|^{2}+|w|^{2}\right) d \theta d \bar{y}+\int_{K}\left(\left|\nabla_{K} \Phi\right|^{2}+|\Phi|^{2}\right) d \bar{y} .
$$

Let $\left|S_{+}^{n}\right|$ denote the volume of $S_{+}^{n}$. Notice that

$$
\int_{S_{+}^{n}}\left(\Theta^{i}\right)^{2} d \theta=\frac{\left|S_{+}^{n}\right|}{n+1} \quad \text { for all } i=1 \cdots n
$$

We define $\varrho_{n}:=\frac{\left|S_{+}^{n}\right|}{n+1}$. 
With these definitions in mind we redefine $\mathbb{L}_{\varepsilon, r}$ by duality as follows

$$
\begin{aligned}
& \int_{(S N K)_{+}} v \mathbb{L}_{\varepsilon, r} v^{\prime} d \theta d \bar{y}:=-\varepsilon^{-2 s} \int_{(S N K)_{+}} \varepsilon^{2} w^{\prime} \Delta_{K} w d \theta d \bar{y} \\
& +\varepsilon^{-2 s} \int_{(S N K)_{+}}\left(\nabla_{S_{+}^{n}} w \nabla_{S_{+}^{n}} w^{\prime}-n w w^{\prime}\right) d \theta d \bar{y} \\
& +\varrho_{n} \int_{K}\left\langle\mathfrak{J} \Phi, \Phi^{\prime}\right\rangle d \bar{y} \\
& +\int_{(S N K)_{+}}\left(\mathcal{J}_{r}^{1}(\Phi)+\mathcal{L}_{r}^{1}(w)+\varepsilon L_{r}(w, \Phi)\right)\left(\varepsilon^{1-2 s} w^{\prime}+\left\langle\Phi^{\prime}, \tilde{\Theta}\right\rangle\right) d \theta d \bar{y} .
\end{aligned}
$$

We associate to $\mathbb{L}_{\varepsilon, r}$ its quadratic bi-linear form

$$
\mathcal{C}_{\varepsilon, r}\left(v, v^{\prime}\right):=\int_{(S N K)_{+}} v \mathbb{L}_{\varepsilon, r} v^{\prime} d \theta d \bar{y},
$$

and the associated quadratic form $\mathcal{Q}_{\varepsilon, r}(v):=\mathcal{C}_{\varepsilon, r}(v, v)$.

As mentioned in the first section, following [11], we want to find the values of $\varepsilon$ for which the operator $\mathbb{L}_{\varepsilon, r}$ is invertible. By selfadjointness this leads to find the values of $\varepsilon$ for which the eigenvalues of the form $\mathcal{Q}_{\varepsilon, r}$ are bounded away from zero. Such techniques requires first that our form should be very close to a model one that we can characterize its spectrum (just the small eigenvalues). Secondly, to understand the behavior of small eigenvalues seeing as "set" valued functions in $\varepsilon$. We will estimate the Morse index of $\mathcal{Q}_{\varepsilon, r}$ and prove the monotonicity of its small eigenvalues. The former can be done using Weyl's asymptotic formula and the latter can be obtained by applying a result by Kato. We shall do this in the remaining of this section.

We define the model form, by duality, as

$$
\begin{aligned}
\mathcal{C}_{0}\left(v, v^{\prime}\right):= & -\varepsilon^{-2 s} \int_{(S N K)_{+}} \varepsilon^{2} w^{\prime} \Delta_{K} w d \theta d \bar{y} \\
& +\varepsilon^{-2 s} \int_{(S N K)_{+}}\left(\nabla_{S_{+}^{n}} w \nabla_{S_{+}^{n}} w^{\prime}-n w w^{\prime}\right) d \theta d \bar{y} \\
& +\varrho_{n} \int_{K}\left\langle\mathfrak{J} \Phi, \Phi^{\prime}\right\rangle d \bar{y}
\end{aligned}
$$

and the associated quadratic form $\mathcal{Q}_{0}(v):=\mathcal{C}_{0}(v, v)$.

Proposition 5.1. There exists a constant $c>0$ (independent of $r$ ) such that

$$
\left|\mathcal{C}_{\varepsilon, r}\left(v, v^{\prime}\right)-\mathcal{C}_{0}\left(v, v^{\prime}\right)\right| \leq c \varepsilon^{s}\|v\|_{H_{\varepsilon}^{1}}\left\|v^{\prime}\right\|_{H_{\varepsilon}^{1}}
$$


Proof. First of all we notice that in $\mathcal{L}_{r}^{1}(w)$ their may appear expressions of the forms $w, \varepsilon \partial_{\bar{y}^{a}} w, \varepsilon^{2} \partial_{\bar{y}^{a}} \partial_{\bar{y}^{b}} w, \partial_{z^{j}} w, \partial_{z^{j}} \partial_{z^{j^{\prime}}} w$. Nevertheless after integrating by parts and using Hölder inequality there holds

$$
\left|\int_{(S N K)_{+}} \varepsilon^{1-2 s} w^{\prime} \mathcal{L}_{r}^{1}(w) d \theta d \bar{y}\right| \leq \varepsilon c\|v\|_{H_{\varepsilon}^{1}}\left\|v^{\prime}\right\|_{H_{\varepsilon}^{1}}
$$

and by definition of the $H_{\varepsilon}^{1}$ norm

$$
\begin{aligned}
\left|\int_{(S N K)_{+}}\left\langle\Phi^{\prime}, \tilde{\Theta}\right\rangle \mathcal{L}_{r}^{1}(w) d \theta d \bar{y}\right| & \leq c \varepsilon^{s}\left\|\varepsilon^{1-2 s} w\right\|_{H_{\varepsilon}^{1}}\left\|\Phi^{\prime}\right\|_{L^{2}(K, N K)} \\
& \leq c \varepsilon^{s}\|v\|_{H_{\varepsilon}^{1}}\left\|v^{\prime}\right\|_{H_{\varepsilon}^{1}} .
\end{aligned}
$$

Furthermore $\Pi \mathcal{J}^{1}(\Phi)=0$. Now it is clear that even if $\mathcal{J}_{r}^{1}(\Phi)+L_{r}(w, \Phi)$ involves terms of the form $w, \varepsilon \partial_{\bar{y}^{a}} w, \varepsilon \partial_{\bar{y}^{a}} \partial_{\bar{y}^{b}} w, \partial_{z^{j}} w, \partial_{z^{j}} \partial_{z^{j}} w$ and also $\Phi^{j}, \partial_{\bar{y}^{a}} \Phi^{j}$ and $\partial_{\bar{y}^{a}} \partial_{\bar{y}^{b}} \Phi^{j}$, in any case after integration by parts and using Hölder inequality we get

$$
\left|\int_{(S N K)_{+}}\left(\varepsilon^{-1} \mathcal{J}_{r}^{1}(\Phi)+L_{r}(w, \Phi)\right)\left(\varepsilon^{1-2 s} w^{\prime}+\left\langle\Phi^{\prime}, \tilde{\Theta}\right\rangle\right) d \theta d \bar{y}\right| \leq c\|v\|_{H_{\varepsilon}^{1}}\left\|v^{\prime}\right\|_{H_{\varepsilon}^{1}} .
$$

The result follows at once.

\section{The Morse index of $\mathcal{Q}_{\varepsilon, r}$}

Define the two quadratic forms

$$
\mathcal{Q}^{ \pm}(v):=\mathcal{Q}_{0}(v) \pm \gamma \varepsilon^{s}\|v\|_{H_{\varepsilon}^{1}}^{2}
$$

From (5.3), if $\gamma>0$ is sufficiently large and $\varepsilon$ small enough, then

$$
\mathcal{Q}^{-} \leq \mathcal{Q}_{\varepsilon, r} \leq \mathcal{Q}^{+}
$$

so that the index of $\mathcal{Q}_{\varepsilon, r}$ is bounded by those of $\mathcal{Q}^{+}$and $\mathcal{Q}^{-}$.

Given any function $w$ defined on $(S N K)_{+}$, we set

$$
\begin{aligned}
D_{0}^{ \pm}(w):= & \left(1 \pm \gamma \varepsilon^{s}\right) \int_{K} \varepsilon^{2}\left|\nabla_{K} w\right|^{2} d \bar{y}-\left(n \mp \gamma \varepsilon^{s}\right) \int_{K}|w|^{2} d \bar{y} \\
D_{1}^{ \pm}(w):= & \left(1 \pm \gamma \varepsilon^{s}\right) \int_{(S N K)_{+}}\left(\varepsilon^{2}\left|\nabla_{K} w\right|^{2}+\left|\nabla_{S_{+}^{n}} w\right|^{2}\right) d \theta d \bar{y} \\
& -\left(n \mp \gamma \varepsilon^{s}\right) \int_{(S N K)_{+}}|w|^{2} d \theta d \bar{y}
\end{aligned}
$$


and finally,

$$
D^{ \pm}(\Phi):=-\left(1 \pm \gamma \varepsilon^{s}\right) \int_{K}\langle\mathfrak{J} \Phi, \Phi\rangle d \bar{y}
$$

With these definitions in mind, we have

$$
\mathcal{Q}^{ \pm}(v)=(n+1) \varrho_{n} \varepsilon^{-2 s} D_{0}^{ \pm}\left(w_{0}\right)+\varepsilon^{-2 s} D_{1}^{ \pm}\left(w_{1}\right)+\varrho_{n} D^{ \pm}(\Phi),
$$

if we decompose $v=\varepsilon^{1-2 s} w+\langle\Phi, \tilde{\Theta}\rangle$ and further decompose $w=w_{0}+w_{1}$ as usual. Following [10, Section 6.3] it is easy to see that if $\left(1 \pm \gamma \varepsilon^{s}\right)>0$ then the index of $D^{ \pm}$is the index of $K$. Moreover the index of $D_{1}^{ \pm}$is equal to zero if $2(n+1)\left(1-\gamma \varepsilon^{s}\right)-\left(n+\gamma \varepsilon^{s}\right)>0$ because

$$
\Pi w_{1}=0 \quad \text { and } \quad \int_{S_{+}^{n}} w_{1} d \theta=0
$$

hence

$$
\int_{S_{+}^{n}}\left|\nabla_{S_{+}^{n}} w_{1}\right|^{2} d \theta \geq 2(n+1) \int_{S_{+}^{n}}\left|w_{1}\right|^{2} d \theta .
$$

This shows that the asymptotic behavior of the index of $\mathcal{Q}_{\varepsilon, r}$ should be determined by $D_{0}^{ \pm}$. It is the case since its index is given by

$$
\sharp\left\{j \quad: \quad\left(1 \pm \gamma \varepsilon^{s}\right) \lambda_{j}<\left(n \mp \gamma \varepsilon^{s}\right)\right\},
$$

where $\lambda_{j}$ are the eigenvalues of $-\varepsilon^{2} \Delta_{K}$ counted with multiplicities. Now using Weyl's formula one obtain its index,

$$
\text { Ind } D_{0}^{ \pm} \sim c_{K}\left(\frac{n}{\varepsilon^{2}}\right)^{\frac{k}{2}}
$$

Collecting these estimates, one obtains the following:

Lemma 5.2. The Morse index of $\mathcal{Q}_{\varepsilon, r}$ is asymptotic to $c \varepsilon^{-k}$ when $\varepsilon$ tends to zero, where $c$ depends only on $m$ and $K$.

\section{Approximate eigenfunctions}

In order to apply Kato's theorem [7] we need to characterize the eigenfunctions (eigenspaces) corresponding to small eigenvalues. We prove:

Lemma 5.3. Let $\sigma$ be an eigenvalue of $\mathbb{L}_{\varepsilon, r}$ and $v=\varepsilon^{1-2 s} w+\langle\Phi, \tilde{\Theta}\rangle$ a corresponding eigenfunction and $\varepsilon^{1-2 s} w_{0}=\int_{S_{+}^{n}} v d \theta$ is the decomposition from (5.2). There exist constants $c, c_{0}>0$ such that if $|\sigma| \leq c_{0}$, then

$$
\left\|v-\varepsilon^{1-2 s} w_{0}\right\|_{H_{\varepsilon}^{1}}^{2} \leq c \varepsilon^{s}\|v\|_{H_{\varepsilon}^{1}}^{2}
$$

for all $\varepsilon>0$ small enough. 
Proof. For any $v^{\prime}=\varepsilon^{1-2 s} w^{\prime}+\left\langle\Phi^{\prime}, \Theta\right\rangle$, we have

$$
\begin{aligned}
\mathcal{C}_{\varepsilon, r}\left(v, v^{\prime}\right) & =\sigma \int_{(S N K)_{+}}\left(\varepsilon^{2-4 s} w w^{\prime}+\langle\Phi, \Theta\rangle\left\langle\Phi^{\prime}, \Theta\right\rangle\right) d \theta d \bar{y} \\
& =\sigma \int_{(S N K)_{+}} \varepsilon^{2-4 s} w w^{\prime} d \theta d \bar{y}+\sigma \varrho_{n} \int_{K}\left\langle\Phi, \Phi^{\prime}\right\rangle d \bar{y}
\end{aligned}
$$

In addition, (5.3) gives

$$
\begin{array}{r}
\mid \int_{(S N K)_{+}} \varepsilon^{-2 s}\left(\varepsilon^{2} \nabla_{K} w \nabla_{K} w^{\prime}+\nabla_{S_{+}^{n}} w \nabla_{S_{+}^{n}} w^{\prime}-\left(n+\sigma \varepsilon^{2-4 s}\right) w w^{\prime}\right) d \theta d \bar{y} \\
+\varrho_{n} \int_{K}\left(\left\langle\mathfrak{J} \Phi, \Phi^{\prime}\right\rangle-\sigma\left\langle\Phi, \Phi^{\prime}\right\rangle\right) d \bar{y} \mid \leq c \varepsilon^{s}\|v\|_{H_{\varepsilon}^{1}}\left\|v^{\prime}\right\|_{H_{\varepsilon}^{1}} .
\end{array}
$$

Step 1. Let $\Phi^{\prime}=0$ and $w^{\prime}=w_{1}$ to get

$$
\begin{aligned}
& \left|\int_{(S N K)_{+}} \varepsilon^{-2 s}\left(\varepsilon^{2}\left|\nabla_{K} w_{1}\right|^{2}+\left|\nabla_{S_{+}^{n}} w_{1}\right|^{2}-\left(n-\sigma \varepsilon^{2-4 s}\right)\left|w_{1}\right|^{2}\right) d \theta d \bar{y}\right| \\
& \leq c \varepsilon^{s}\|v\|_{H_{\varepsilon}^{1}}\left\|\varepsilon^{1-2 s} w_{1}\right\|_{H_{\varepsilon}^{1}} .
\end{aligned}
$$

However, since

$$
\Pi w_{1}=0 \quad \text { and } \quad \int_{S_{+}^{n}} w_{1} d \theta=0
$$

we have

$$
\int_{S_{+}^{n}}\left|\nabla_{S_{+}^{n}} w_{1}\right|^{2} d v o l_{S_{+}^{n}} \geq 2(n+1) \int_{S_{+}^{n}}\left|w_{1}\right|^{2} d \theta
$$

hence

$$
\begin{aligned}
& \left|\int_{(S N K)_{+}} \varepsilon^{-2 s}\left(\varepsilon^{2}\left|\nabla_{K} w_{1}\right|^{2}+\frac{1}{2}\left|\nabla_{S_{+}^{n}} w_{1}\right|^{2}+\left(1-|\sigma| \varepsilon^{2-4 s}\right)\left|w_{1}\right|^{2}\right) d \theta d \bar{y}\right| \\
& \leq c \varepsilon^{s}\|v\|_{H_{\varepsilon}^{1}}^{2} .
\end{aligned}
$$

This implies that

$$
\left\|\varepsilon^{1-2 s} w_{1}\right\|_{H_{\varepsilon}^{1}}^{2} \leq c \varepsilon^{s}\|v\|_{H_{\varepsilon}^{1}}^{2},
$$

for all $\varepsilon \in(0,1)$, provided $|\sigma| \leq 1 / 2$.

Step 2. Now let $w^{\prime}=0$ and $\Phi^{\prime}=\Phi^{+}$(respectively $\Phi^{\prime}=\Phi^{-}$) in (5.4), where $\Phi^{+}$ (respectively $\Phi^{-}$) is the $L^{2}$ projection of $\Phi$ over the space of eigenfunctions of $\mathfrak{J}$ associated to positive (respectively negative) eigenvalues. This yields

$$
\left|\int_{K}\left(\left\langle\mathcal{J} \Phi, \Phi^{ \pm}\right\rangle-\sigma\left\langle\Phi, \Phi^{ \pm}\right\rangle\right) d \bar{y}\right| \leq c \varepsilon^{s}\|v\|_{H_{\varepsilon}^{1}}\left\|\left\langle\Phi^{ \pm}, \tilde{\Theta}\right\rangle\right\|_{H_{\varepsilon}^{1}} .
$$


Since $\mathfrak{J}$ is invertible, there exists $c_{1}>0$ such that

$$
c_{1}\left\|\left\langle\Phi^{ \pm}, \tilde{\Theta}\right\rangle\right\|_{H_{\varepsilon}^{1}}^{2} \leq\left|\int_{K}\left\langle\mathfrak{J} \Phi, \Phi^{ \pm}\right\rangle d \bar{y}\right| .
$$

Hence

$$
\left(c_{1}-|\sigma|\right)\left\|\left\langle\Phi^{ \pm}, \tilde{\Theta}\right\rangle\right\|_{H_{\varepsilon}^{1}}^{2} \leq c \varepsilon^{s}\|v\|_{H_{\varepsilon}^{1}}^{2} .
$$

This conclude the proof with $c_{0}:=\min \left\{1 / 2, c_{1} / 2\right\}$.

Remark 5.4. If $v$ is an eigenspace corresponding to an eigenvalue given by the above lemma, then it satisfies

$$
\begin{aligned}
\mid \int_{(S N K)_{+}} \varepsilon^{-2 s}\left(\varepsilon^{2}\left|\nabla_{K} w\right|^{2}\right. & \left.+\left|\nabla_{S_{+}^{n}} w\right|^{2}-\left(n+\sigma \varepsilon^{2-4 s}\right)|w|^{2}\right) d \theta d \bar{y} \\
& +\varrho_{n} \int_{K}(\langle\mathfrak{J} \Phi, \Phi\rangle-\sigma\langle\Phi, \Phi\rangle) d \bar{y} \mid \leq c \varepsilon^{s}\|v\|_{H_{\varepsilon}^{1}}^{2}
\end{aligned}
$$

and

$$
\left|\int_{(S N K)_{+}} \varepsilon^{-2 s}\left(\varepsilon^{2}\left|\nabla_{K} w\right|^{2}+\left|\nabla_{S_{+}^{n}} w\right|^{2}-n|w|^{2}\right) d \theta d \bar{y}\right| \leq c \varepsilon^{s}\|v\|_{H_{\varepsilon}^{1}}^{2} .
$$

Notice that $\nabla_{S_{+}^{n}} w=\nabla_{S_{+}^{n}} w_{1}$ if $w$ is decomposed as $w=w_{0}+w_{1}$ one has

$$
\left|\int_{(S N K)_{+}} \varepsilon^{-2 s}\left(\varepsilon^{2}\left|\nabla_{K} w\right|^{2}-n|w|^{2}\right) d \theta d \bar{y}\right| \leq c \varepsilon^{s}\|v\|_{H_{\varepsilon}^{1}}^{2}
$$

so that

$$
\varepsilon^{-2 s} \int_{(S N K)_{+}} \varepsilon^{2}\left|\nabla_{K} w\right|^{2} d \theta d \bar{y} \leq c \varepsilon^{s}\|v\|_{H_{\varepsilon}^{1}}^{2}+n \varepsilon^{-2 s} \int_{(S N K)_{+}}|w|^{2} d \theta d \bar{y} .
$$

In particular we have

$$
\|v\|_{H_{\varepsilon}^{1}} \leq c\|v\|_{L_{\varepsilon}^{2}}
$$

\section{Variation of small eigenvalues with respect to $\varepsilon$}

To understand the behavior of small eigenvalues of the symmetric quadratic form $\mathcal{Q}_{\varepsilon, r}$, we need to apply a result by Kato, see [7]. Considering the eigenvalues $\sigma(\varepsilon)$ as differentiable multivalued function in $\varepsilon$, the result states that

$$
\partial_{\varepsilon} \sigma \in\left\{\int_{(S N K)_{+}} v\left(\partial_{\varepsilon} \mathbb{L}_{\varepsilon, r}\right) v d \theta d \bar{y} \quad: \quad \mathbb{L}_{\varepsilon, r} v=\sigma v, \quad\|v\|_{L^{2}}=1\right\} .
$$

An good estimate of a bound for the set on the right of (5.6) allows one to estimate the spectral gaps of the linearized operator when the parameter $\varepsilon$ is small, see [10, Section 6.3].

This is indeed given in the following lemma. 
Lemma 5.5. There exist constants $c_{1}, c>0$ such that, if $\sigma$ is an eigenvalue of $\mathbb{L}_{\varepsilon, r}$ with $|\sigma|<c_{1}$, then

$$
\varepsilon \partial_{\varepsilon} \sigma \geq 2 n-c \varepsilon^{s},
$$

provided $\varepsilon$ is small enough.

Proof. We have just to provide bounds for the set on the right of (5.6) using the above remark.

Assume that $\mathbb{L}_{\varepsilon, r} v=\sigma v$, but rather than normalizing the function $v$ by $\|v\|_{L^{2}}=1$, assume instead that $\|v\|_{L_{\varepsilon}^{2}}=1$. In order to compute $\partial_{\varepsilon} \mathbb{L}_{\varepsilon, r}$, recall that

$$
w=\varepsilon^{-1+2 s} \Pi^{\perp} v \quad \text { and that } \quad\langle\mathfrak{J} \Phi, \tilde{\Theta}\rangle=\Pi v,
$$

so we can write

$$
\begin{aligned}
\mathbb{L}_{\varepsilon, r} v= & -\varepsilon^{2 s} \Delta_{K}\left(\Pi^{\perp} v\right)+\frac{1}{\varepsilon^{2-2 s}} \mathcal{L}_{0}\left(\Pi^{\perp} v\right)+\Pi v+\frac{1}{\varepsilon^{1-2 s}} \mathcal{L}_{r}^{1}\left(\Pi^{\perp} v\right) \\
& +\mathcal{J}^{1}\left(\mathfrak{J}_{r}^{-1} \Pi v\right)+\varepsilon L_{r}\left(\varepsilon^{-1+2 s} \Pi^{\perp} v, \mathfrak{J}^{-1} \Pi v\right) .
\end{aligned}
$$

Since $\Pi$ and $\Pi^{\perp}$ are independent of $\varepsilon$, we have

$$
\begin{aligned}
\partial_{\varepsilon} \mathbb{L}_{\varepsilon, r} v= & -2 s \varepsilon^{-1+2 s} \Delta_{K}\left(\Pi^{\perp} v\right)+(-2+2 s) \varepsilon^{-3+2 s} \mathcal{L}_{0}\left(\Pi^{\perp} v\right) \\
& +(-1+2 s) \varepsilon^{-2+2 s} \mathcal{L}_{r}^{1}\left(\Pi^{\perp} v\right)+\tilde{L}_{r}\left(\varepsilon^{-1+2 s} \Pi^{\perp} v, \mathfrak{J}^{-1} \Pi v\right),
\end{aligned}
$$

where the operator $\tilde{L}_{r}$ varies from line to line but satisfies the usual assumptions. This now gives

$$
\begin{aligned}
& \left.\quad\left|\int_{(S N K)_{+}} v\left(\partial_{\varepsilon} \mathbb{L}_{\varepsilon, r}\right) v d \theta d \bar{y}-2 \varepsilon^{-1-2 s} \int_{(S N K)_{+}} \varepsilon^{2}\right| \nabla_{K} w\right|^{2} d \theta d \bar{y} \\
& \quad+\frac{(2-2 s)}{\varepsilon} \varepsilon^{-2 s} \int_{(S N K)_{+}}\left(\varepsilon^{2}\left|\nabla_{K} w\right|^{2}+\left|\nabla_{S_{+}^{n}} w\right|^{2}-n|w|^{2}\right) d \theta d \bar{y} \mid \\
& \leq c\|v\|_{H_{\varepsilon}^{1}}^{2}+\left|\frac{1-2 s}{\varepsilon} \int_{(S N K)_{+}}\langle\Phi, \tilde{\Theta}\rangle \mathcal{L}_{r}^{1}(w) d \theta d \bar{y}\right| \\
& \leq \frac{c}{\varepsilon^{1-s}}\|v\|_{H_{\varepsilon}^{1}}^{2} .
\end{aligned}
$$

Consequently if $v$ is an eigenfunction of $\mathbb{L}_{\varepsilon, r}$ with corresponding eigenvalue $|\sigma| \leq$ $c_{0}$, where $c_{0}$ is given in the previous lemma, by the inequality (5.5), see the above remark, we have

$$
\left.\left|\int_{(S N K)_{+}} v\left(\partial_{\varepsilon} \mathbb{L}_{\varepsilon, r}\right) v d \theta d \bar{y}-2 \varepsilon^{-1-2 s} \int_{(S N K)_{+}} \varepsilon^{2}\right| \nabla_{K} w\right|^{2} d \theta d \bar{y} \mid \leq \frac{c}{\varepsilon^{1-s}}\|v\|_{H_{\varepsilon}^{1}}^{2} .
$$


Again from the above remark, one gets

$\varepsilon^{-1-2 s} \int_{(S N K)_{+}} \varepsilon^{2}\left|\nabla_{K} w\right|^{2} d \theta d \bar{y} \leq c \varepsilon^{-1+s}\|v\|_{H_{\varepsilon}^{1}}^{2}+n \varepsilon^{-1-2 s} \int_{(S N K)_{+}}|w|^{2} d \theta d \bar{y}$.

If we normalize $v$ by $\|v\|_{L_{\varepsilon}^{2}}=1$ then inserting this into (5.7) we get

$$
\left|\int_{(S N K)_{+}} v\left(\partial_{\varepsilon} \mathbb{L}_{\varepsilon, r}\right) v d \theta d \bar{y}-\frac{2}{\varepsilon} n\right| \leq \frac{c}{\varepsilon^{1-s}}
$$

for all eigenfunction $v$ such that $\mathbb{L}_{\varepsilon, r} v=\sigma v$ which is normalized by $\|v\|_{L_{\varepsilon}^{2}}=1$.

Now since $\|v\|_{L^{2}} \leq\|v\|_{L_{\varepsilon}^{2}}$, we conclude that

$$
\inf _{\substack{\mathbb{L}_{\varepsilon} v=\sigma v \\\|v\|_{L^{2}}=1}} \int_{(S N K)_{+}} v\left(\partial_{\varepsilon} \mathbb{L}_{\varepsilon}\right) v d \theta d \bar{y} \geq \inf _{\substack{\mathbb{L}_{\varepsilon} v=\sigma v \\\|v\|_{L_{\varepsilon}^{2}}=1}} \int_{(S N K)_{+}} v\left(\partial_{\varepsilon} \mathbb{L}_{\varepsilon}\right) v d \theta d \bar{y}
$$

and (5.8) implies that

$$
\partial_{\varepsilon} \sigma \geq \frac{2}{\varepsilon} n-\frac{c}{\varepsilon^{1-s}} .
$$

This completes the proof of the result.

\section{Proof of Theorem 1.1}

Using Lemma 5.2 and Lemma 5.5, reasoning as for the proof of [10, Lemma 6.3] we can find a sequence of open interval $I_{i}, i \in \mathbb{N}$ such that the smallest eigenvalue of $\mathbb{L}_{\varepsilon, r}$ is bounded away from zero for any $\varepsilon \in \cup_{i} I_{i}$. More precisely we have:

Lemma 6.1. Fix any $q \geq 2$. Then there exists a sequence of disjoint nonempty open intervals $I_{i}=\left(\varepsilon_{i}^{-}, \varepsilon_{i}^{+}\right), \varepsilon_{i}^{ \pm} \rightarrow 0$ and a constant $c_{q}>0$ such that when $\varepsilon \in I^{q}:=\cup_{i} I_{i}$, the operator $\mathbb{L}_{\varepsilon, r}$ is invertible and

$$
\left(\mathbb{L}_{\varepsilon, r}\right)^{-1}: L_{\varepsilon}^{2} \longrightarrow L_{\varepsilon}^{2},
$$

has norm bounded by $c_{q} \varepsilon^{-k-q+1}$, uniformly in $\varepsilon \in I$. Furthermore, $I^{q}:=\cup_{i} I_{i}$ satisfies

$$
\left|\mathcal{H}^{1}\left((0, \varepsilon) \cap I^{q}\right)-\varepsilon\right| \leq c \varepsilon^{q}, \quad \varepsilon \searrow 0
$$

For $p \in \mathbb{N}$ and $0<\alpha<1$, we denote by $\mathcal{C}^{p, \alpha}$ the usual Hölder spaces on the closure of $(S N K)_{+}$. 
Lemma 6.2. Let $f \in \mathcal{C}^{0, \alpha}$ and $v$ satisfy

$$
\mathbb{L}_{\varepsilon, r} v=f
$$

Then there exit a constant $c>0$ (independent of $\varepsilon$ but depend on $r$ ) and $R>0$ depending only on $q, \alpha$, $s$ and $k$ such that

$$
\|v\|_{\mathcal{C}^{2, \alpha}} \leq c \varepsilon^{-R}\|f\|_{\mathcal{C}^{0, \alpha}}
$$

for any $\varepsilon \in I^{q}$.

Proof. Fix $q \geq 2$. Observe that by definition of the weighted norm of $L_{\varepsilon}^{2}$, from Lemma 6.1 we have

$$
\|v\|_{L^{2}} \leq c_{q} \varepsilon^{-k-q+1-s}\|f\|_{L^{2}} .
$$

By standard elliptic regularity theory, there exists $c>0$ (depending on $r$ ) such that the following Hölder estimate holds

$$
\varepsilon^{2+\alpha}\|v\|_{\mathcal{C}^{2, \alpha}} \leq c \varepsilon^{2}\|f\|_{\mathcal{C}^{0, \alpha}}+c \varepsilon^{-\frac{k}{2}}\|v\|_{L^{2}}
$$

From these last two inequalities, we can choose $R>\frac{3 k}{2}+q+\alpha+1+s$.

We end the proof of the main theorem by finding a fixed point for the mapping

$$
T_{\varepsilon, r}(v):=-\left(\mathbb{L}_{\varepsilon, r}\right)^{-1}\left\{\mathcal{O}_{r}\left(\varepsilon^{r}\right)+\mathcal{N}_{\varepsilon, r}(v)\right\}
$$

where

$$
\begin{aligned}
\int_{(S N K)_{+}} \mathcal{N}_{\varepsilon, r}(v) v^{\prime} d \theta d \bar{y}:= & \int_{(S N K)_{+}} \varepsilon^{-1} Q_{r}\left(\varepsilon^{-1+2 s} \Pi^{\perp} v, \Pi v\right) v^{\prime} d \theta d \bar{y} \\
& +\oint_{\partial(S N K)_{+}} \varepsilon^{-1} \bar{Q}_{r}\left(\varepsilon^{-1+2 s} \Pi^{\perp} v, \Pi v\right) v^{\prime} d \bar{\theta} d \bar{y}
\end{aligned}
$$

Since by definition, $Q_{r}$ and $\bar{Q}_{r}$ are (at least) quadratic we have

$$
\begin{gathered}
\left\|\mathcal{N}_{\varepsilon, r}(v)\right\|_{\mathcal{C}^{0, \alpha}}=\varepsilon^{-2+2 s} O\left(\|v\|_{\mathcal{C}^{2, \alpha}}\right)\|v\|_{\mathcal{C}^{2, \alpha}}^{2} ; \\
\left\|\mathcal{N}_{\varepsilon, r}\left(v_{1}\right)-\mathcal{N}_{\varepsilon, r}\left(v_{2}\right)\right\|_{\mathcal{C}^{0, \alpha}}=\varepsilon^{-2+2 s} O\left(\left\|v_{1}\right\|_{\mathcal{C}^{2, \alpha}},\left\|v_{2}\right\|_{\mathcal{C}^{2, \alpha}}\right)\left\|v_{1}-v_{2}\right\|_{\mathcal{C}^{2, \alpha}} .
\end{gathered}
$$

Now we fix $r>2 R+2-2 s$. By Lemma 6.2 and the above inequalities, for every $\varepsilon \in I^{q}, T_{\varepsilon, r}(v)$ maps the ball

$$
\left\{v \in \mathcal{C}^{2, \alpha} \quad: \quad\|v\|_{\mathcal{C}^{2, \alpha}} \leq C \varepsilon^{r+1-R}\right\}
$$


into itself moreover it is a contraction. Therefore it has a unique fixed point $v=$ $\varepsilon^{1-2 s} w+\langle\Phi, \tilde{\Theta}\rangle$ in the ball yielding

$$
\begin{aligned}
& m H\left(\hat{w}^{(r)}+w, \hat{\Phi}^{r}+\Phi\right)=n \quad \text { in } \quad S_{\varepsilon}\left(\hat{w}^{(r)}+w, \hat{\Phi}^{r}+\Phi\right) \subset \Omega_{\varepsilon}, \\
& \left\langle N, \mathcal{V}^{\varepsilon}\right\rangle=0 \quad \text { on } \quad \partial S_{\varepsilon}\left(\hat{w}^{(r)}+w, \hat{\Phi}^{r}+\Phi\right) \subset \partial \Omega_{\varepsilon} .
\end{aligned}
$$

If $\varepsilon \in I^{q}$ is sufficiently small then rescaling back, the tube $\varepsilon S_{\varepsilon}\left(\hat{w}^{(r)}+w, \hat{\Phi}^{r}+\Phi\right)$, is an embedded hypersurface of $\Omega$ with constant mean curvature equal to $\frac{n}{m} \varepsilon^{-1}$ and intersecting the boundary of $\Omega$ perpendicularly along its boundary.

Remark 6.3 (Existence of stationary capillary hypersurfaces). Letting $\gamma \in(0, \pi)$ be an angle, recall from Subsection 2.1 that $\left(y^{1}, y^{2} \ldots, y^{k}\right) \in \mathbb{R}^{k}$ (respectively $\left.\left(z^{1}, z^{2} \ldots, z^{n}\right) \in B_{r(\gamma)}^{n}\right)$ are the local coordinate variables on $K_{\varepsilon}$ (respectively on $S^{n}(\gamma)$ ), where $r(\gamma):=\frac{1-\cos \gamma}{1+\cos (\gamma)}$ (see Subsection 2.4) and

$$
\Theta(\gamma):=\left.\mathbf{p}\right|_{B_{r(\gamma)}^{n}}-\cos (\gamma) E_{n+1}
$$

parameterizes the spherical cap $S^{n}(\gamma)$ which intersects the horizontal plane $\mathbb{R}^{m}$ with angle $\gamma$.

As in the case where $\gamma=\frac{\pi}{2}$, we can use the same class of deformations letting $\Phi: K \rightarrow N K_{\varepsilon}$ and $w: B_{\gamma}^{n} \times K_{\varepsilon} \rightarrow \mathbb{R}$, consider

$$
S_{\gamma}:(y, z) \mapsto y \times \varepsilon^{-1} \Phi(\varepsilon y)+(1+w(y, z)) \Theta(\gamma) .
$$

The surfaces nearby a geodesic tube around $K_{\varepsilon}$ which make an angle almost equal to $\gamma$ with $\partial \Omega_{\varepsilon}$ can be parameterized (locally) by

$$
G_{\gamma}(y, z):(y, z) \longrightarrow S_{\gamma}(y, z) \longrightarrow F^{\varepsilon}\left(S_{\gamma}(y, z)\right),
$$

namely

$$
G_{\gamma}(y, z):=F^{\varepsilon}\left(y, \frac{1}{\varepsilon} \Phi(\varepsilon y)+(1+w(y, z)) \tilde{\Theta}(\gamma),(1+w(y, z)) \Theta^{n+1}(\gamma)\right) .
$$

Notice that $\left.\Theta^{n+1}(\gamma)\right|_{\partial B_{r(\gamma)}^{n}}=0$, so there holds

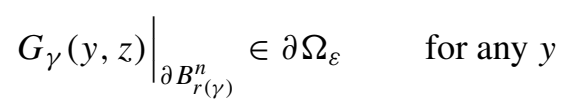

The image of this map will be called $S_{\varepsilon}^{\gamma}(w, \Phi)$.

Observe that the hypersurfaces close to $S_{\varepsilon}^{\gamma}(0,0)$ are parameterized using the vector field $-\Upsilon(\gamma)=\Theta^{j}(\gamma) X_{j}+\Theta^{n+1}(\gamma) \mathcal{V}^{\varepsilon}$ rather than the normal $\boldsymbol{\Xi}:=\mathbf{p}^{j} X_{j}+$ 
$\mathbf{p}^{n+1} \mathcal{V}^{\varepsilon}$ because it is more reasonable if we want the boundary of $S_{\varepsilon}^{\gamma}(w, \Phi)$ to be on $\partial \Omega_{\varepsilon}$ without imposing simultaneously a Neumann and Dirichlet boundary condition on $w$. Suppose $Z_{j}(\gamma), Z_{a}(\gamma)$ span the tangent space of $S_{\varepsilon}^{\gamma}(w, \Phi)$ as in Paragraph 3.0.3, we can obtain the normal fields $N(\gamma)$ by finding $\alpha^{j}(\gamma)$ and $\beta^{a}(\gamma)$ so that

$$
N(\gamma)=-\Xi+\alpha^{j}(\gamma) Z_{j}(\gamma)+\beta^{a}(\gamma) Z_{a}(\gamma)
$$

As we did in Section 3, the mean curvature at every point of $S_{\varepsilon}^{\gamma}(w, \Phi)$ can be obtained:

$$
\begin{aligned}
& m H(w, \Phi) \\
= & n-\varepsilon\left(\Gamma_{a}^{a}(\tilde{\mathbf{p}})+\mathbf{p}^{n+1} h_{a a}+\mathbf{p}^{n+1}\left[3\langle h(\tilde{\mathbf{p}}), \tilde{\mathbf{p}}\rangle-h_{j j}\right]+n \Theta^{n+1}(\gamma)\langle h(\tilde{\mathbf{p}}), \tilde{\mathbf{p}}\rangle\right) \\
& +\mathcal{O}\left(\varepsilon^{2}\right)-\left(\varepsilon^{2} \Delta_{K}(\langle\Theta(\gamma), \mathbf{p}\rangle w)+\Delta_{S^{n}}(\langle\Theta(\gamma), \mathbf{p}\rangle w)+n(\langle\Theta(\gamma), \mathbf{p}\rangle w)\right) \\
& -\varepsilon\left(\left\langle\Delta_{K} \Phi+R\left(\Phi, E_{a}\right) E_{a}, \tilde{\mathbf{p}}\right\rangle-\Gamma_{a}^{c}(\Phi) \Gamma_{c}^{a}(\tilde{\mathbf{p}})\right) \\
& -\varepsilon\left((3 n+1) \Theta^{n+1}(\gamma) h(\tilde{\mathbf{p}})^{a}\left\langle\Phi_{\bar{a}}, \tilde{\mathbf{p}}\right\rangle+\mathbf{p}^{n+1} h\left(\Phi_{\bar{a}}\right)^{a}+2 \mathbf{p}^{n+1} h: \Gamma(\Phi)\right) \\
& -\frac{n}{2}\left(\varepsilon w_{\bar{a}}+\left\langle\Phi_{\bar{a}}, \tilde{\mathbf{p}}\right\rangle\right)^{2}-\left\langle\Phi_{\bar{a}}, \varepsilon \nabla_{S^{n}} w_{\bar{a}}\right\rangle-2 \varepsilon^{2} \nabla_{K}^{2} w: \Gamma(\Phi) \\
& +\frac{n+2}{6}\langle R(\Phi, \tilde{\mathbf{p}}) \Phi, \tilde{\mathbf{p}}\rangle-\frac{1}{3}\left\langle R\left(\Phi, E_{i}\right) \Phi, E_{i}\right\rangle \\
& +\varepsilon L(w)+\varepsilon^{2} L(w, \Phi)+Q(w)+\varepsilon Q(w, \Phi) .
\end{aligned}
$$

Moreover (recall that $\mathcal{V}^{\varepsilon}$ is the interior normal of $\partial \Omega_{\varepsilon}$ ) using the fact that $\left.\Theta^{n+1}(\gamma)\right|_{\partial B_{r(\gamma)}^{n}}=0$, the equation $\left\langle-\mathcal{V}^{\varepsilon}, N\right\rangle=\cos (\gamma)$ is equivalent to

$$
\begin{aligned}
\langle\Theta(\gamma), \mathbf{p}\rangle(1-w) \frac{\partial w}{\partial \eta(\gamma)}= & \overline{\mathcal{O}}\left(\varepsilon^{2}\right)+\varepsilon^{2} \bar{L}(w, \Phi) \\
& +\bar{Q}^{1}(w, \Phi)+\varepsilon \bar{Q}(w, \Phi) \quad \text { on } \partial S^{n}(\gamma) \times K,
\end{aligned}
$$

which is again equivalent to

$$
\begin{aligned}
& \frac{\partial(\langle\Theta(\gamma), \mathbf{p}\rangle w)}{\partial \eta(\gamma)}=w \frac{\partial\langle\Theta(\gamma), \mathbf{p}\rangle}{\partial \eta(\gamma)}+\overline{\mathcal{O}}\left(\varepsilon^{2}\right)+\varepsilon^{2} \bar{L}(w, \Phi)+\bar{Q}^{1}(w, \Phi)+\bar{Q}(w) \\
& +\varepsilon \bar{Q}(w, \Phi) \quad \text { on } \partial S^{n}(\gamma) \times K \\
& =w \cot (\gamma)+\overline{\mathcal{O}}\left(\varepsilon^{2}\right)+\varepsilon^{2} \bar{L}(w, \Phi)+\bar{Q}^{1}(w, \Phi) \\
& +\bar{Q}(w)+\varepsilon \bar{Q}(w, \Phi) \\
& \text { on } \partial S^{n}(\gamma) \times K \text {, }
\end{aligned}
$$


where

$$
\bar{Q}^{1}(w, \Phi):=\cot (\gamma)\left(\varepsilon w_{\bar{a}}\left\langle\Phi_{\bar{a}}, \tilde{\mathbf{p}}\right\rangle+\left\langle\Phi_{\bar{a}}, \tilde{\mathbf{p}}\right\rangle\left\langle\Phi_{\bar{a}}, \tilde{\mathbf{p}}\right\rangle-\frac{1}{3}\langle R(\Phi, \tilde{\mathbf{p}}) \Phi, \tilde{\mathbf{p}}\rangle\right) .
$$

Using the results from Section 2.4 and from Section 4, one can adjust the tube to $S_{\varepsilon}^{\gamma}\left(\hat{w}^{(r)}, \hat{\Phi}^{(r)}\right)$ accurately. Moreover with the decomposition of the functions $v=\varepsilon^{1-2 s} w+\langle\Phi, \tilde{\mathbf{p}}\rangle \in L^{2}\left(S^{n}(\gamma) \times K\right)$ as in (5.1) we conclude that the spectral analysis of the linearized mean curvature operator over $S_{\varepsilon}^{\gamma}\left(\hat{w}^{(r)}, \hat{\Phi}^{(r)}\right)$ carried out as we obtain in Section 5 in the new weighted Hilbert subspaces of $L^{2}\left(S^{n}(\gamma) \times K\right)$

$$
\begin{gathered}
L_{\varepsilon, \gamma}^{2}:=\left\{v=\varepsilon^{1-2 s} w+\langle\Phi, \tilde{\mathbf{p}}\rangle \in L^{2}\left(S^{n}(\gamma) \times K\right) \quad:\right. \\
\left.\varepsilon^{-2 s} \int_{S^{n}(\gamma) \times K}\langle\Theta(\gamma), \mathbf{p}\rangle|w|^{2} d \theta(\gamma) d \bar{y}+\int_{K}|\Phi|^{2} d \bar{y}<\infty\right\} \\
\left\{v \in L_{\varepsilon, \gamma}^{2}: \varepsilon^{-2 s} \int_{S^{n}(\gamma) \times K}\langle\Theta(\gamma), \mathbf{p}\rangle\left(\varepsilon^{2}\left|\nabla_{K} w\right|^{2}+\left|\nabla_{S^{n}(\gamma)} w\right|^{2}+|w|^{2}\right) d \theta(\gamma) d \bar{y}\right. \\
\left.+\int_{K}\left(\left|\nabla_{K} \Phi\right|^{2}+|\Phi|^{2}\right) d \bar{y}<\infty\right\} .
\end{gathered}
$$

Under the usual assumptions on $K$, if $\varepsilon \in I^{q}$ is sufficiently small then rescaling back, we can find a couple $(w, \Phi)$ so that the tube $\varepsilon S_{\varepsilon}^{\gamma}\left(\hat{w}^{(r)}+w, \hat{\Phi}^{r}+\Phi\right)$, is an embedded hypersurface of $\Omega$ with constant mean curvature $\frac{n}{m} \varepsilon^{-1}$ and intersecting $\partial \Omega$ with and angle $\gamma$. This yields a set of stationary capillary hypersurfaces in $\Omega$ with constant "contact angle" $\gamma$ and condensing to the submanifold $K$.

\section{References}

[1] W. BÜRGER and E. KUWERT, Area-minimizing disks with free boundary and prescribed enclosed volume, J. Reine Angew. Math., to appear.

[2] M. M. FALL, Embedded disc-type surfaces with large constant mean curvature and free boundaries, Commun. Contemp. Math., to appear.

[3] R. FInN, "Equilibrium Capillary Surfaces", Springer-Verlag, New York, 1986.

[4] M. GrÜter and J. Jost, On embedded minimal discs in convex bodies, Ann. Inst. H. Poincaré, Anal. Non Linéaire 3 (1986), 345-390.

[5] G. HUISKEN and S. T. YAU, Definition of center of mass for isolated physical systems and unique foliations by stable spheres with constant mean curvature, Invent. Math. 124 (1996), 281-311.

[6] J. Jost, Existence results for embedded minimal surfaces of controlled topological type I, Ann. Scuola Norm. Sup. Pisa. Cl. Sci. (4) 13 (1986), 15-50.

[7] T. Kato, "Perturbation Theory for Linear Operators", GMW 132, Springer-Verlag, 1976.

[8] H. B. Lawson, Complete minimal surfaces in $S^{3}$, Ann. of Math. (2) 92 (1970), 335-374.

[9] H. B. LAWSON, "Lectures on Minimal Submanifolds", Vol. I, second edition, Mathematics Lecture Series, 9, Publish or Perish, Wimington, Del., 1980. 
[10] F. Mahmoudi, R. MAZZeo and F. PACARD, Constant mean curvature hypersurfaces condensing along a submanifold, Geom. Funct. Anal. 16 (2006), 924-958.

[11] F. MAHMOUDI and A. MALCHIODI, Concentration on minimal submanifolds for a singularly perturbed Neumann problem, Adv. Math. 209 (2007), 460-525.

[12] A. MALCHIODI, Concentration at curves for a singularly perturbed Neumann problem in three-dimensional domains, Geom. Funct. Anal., 15 (2005), 1162-1222.

[13] A. MALChIODI and M. Montenegro, Boundary concentration phenomena for a singularly perturbed elliptic problem, Comm. Pure Appl. Math. 55 (2002), 1507-1568.

[14] A. Malchiodi and M. Montenegro, Multidimensional Boundary-layers for a singularly perturbed Neumann problem, Duke Math. J. 124 (2004), 105-143.

[15] R. MAZZEO and F. PACARD, Foliations by constant mean curvature tubes, Comm. Anal. Geom. 13 (2005), 633-670.

[16] M. RitorÉ and C. Ros ALES, Existence and charaterization of regions minimizing perimeter under a volume constraint inside Euclidean cones, Trans. Amer. Math. Soc. 356, 46014622.

[17] A. Ros, "The Isoperimetric Problem", Lecture series given during the Caley Mathematics Institute Summer School on the Global Theory of Minimal Surfaces at the MSRI, Berkley, California, 2001.

[18] A. Ros and R. SouAm, On stability of capillary surfaces in a ball, Pacific J. Math. 178 (1997), 345-361.

[19] A. Ros and E. Vergasta, Stability for hypersurfaces of constant mean curvature with free boundary, Geom. Dedicata 56 (1995), 19-33.

[20] R. SCHOEN and S. T. YAU, Existence of incompressible minimal surfaces and the topology of three-dimensional manifolds with nonnegative scalar curvature, Ann. of Math. (2) 110 (1979), 127-142.

[21] P. Sternberg and K. Zumbrun, Connectivity of phase boundaries in strictly convex domains, Arch. Ration. Mech. Anal. 141 (1998), 375-400.

[22] M. STRUWE, Non-uniqueness in the Plateau problem for surfaces of constant mean curvature, Arch. Ration. Mech. Anal. 93 (1986), 135-157.

[23] M. STRUWE, The existence of surfaces of constant mean curvature with free boundaries, Acta Math. 160 (1988), 19-64.

[24] M. STRUwe, On a free boundary problem for minimal surfaces, Invent. Math. 75 (1984), 547-560.

[25] R. YE, Foliation by constant mean curvature spheres, Pacific J. Math. 147 (1991), 381-396.

[26] T. J. Willmore, "Riemannian Geometry", Oxford Univ. Press. NY., 1993.

SISSA

Sector of Mathematical Analysis

Via Beirut 2-4

34014 Trieste, Italy

fall@sissa.it

Département de Matématiques

Faculté des sciences de Tunis

Campus Universitaire

2092 Tunis, Tunisia

mahmoudi@sissa.it 